\title{
BIFURCATION AND SYMMETRY BREAKING IN APPLIED MATHEMATICS
}

\author{
BY D. H. SATTINGER ${ }^{1}$
}

I. Introduction. Bifurcation theory is the study of branch points in nonlinear equations, that is, of singular points of the equations where several solutions come together. It is important in applications because bifurcation phenomena typically accompany the transition to instability when a characteristic parameter passes through a critical value. To state the situation more precisely, suppose the states of a physical system are determined as solutions of a functional equation

$$
G(\lambda, u)=0
$$

where $\lambda \in \Lambda$ is a parameter, $u$ is an element of a Banach space $\mathcal{E}$, and $G$ is a mapping from $\Lambda \times \mathcal{E}$ to another Banach space $\mathcal{F}$. Let $\mathcal{S}$ be the zero set of $G$ in $\Lambda \times \mathcal{E}$ and suppose $\gamma$ is a smooth curve in $\delta$. Then a branch point $\left(\lambda_{c}, u_{c}\right)$ is a point of $\gamma$ such that for any neighborhood of $U$ of $\left(\lambda_{c}, u_{c}\right)$ in $\Lambda \times \mathcal{E}$, $(U \backslash \gamma) \cap \mathcal{S} \neq \varnothing$. Some typical "bifurcation diagrams" are pictured schematically in Figure 1.

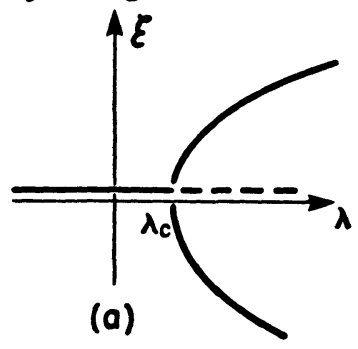

(a)

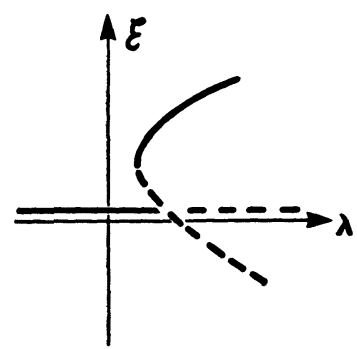

(b)

FIGURE 1.

Schematic diagram of bifurcation at $\left(\lambda_{c}, 0\right)$; one nontrivial branch. The vertical axis represents a Banach space $\mathcal{E}$.

Closely tied to the phenomenon of bifurcation is the property of stability. Suppose the solutions of (1) represent equilibrium solutions for a dynamical system which evolves according to the time dependent equations $u_{t}=G(\lambda, u)$. An equilibrium solution $u_{0}$ is stable if small perturbations from it remain close to $u_{0}$ as $t \rightarrow \infty ; u_{0}$ is asymptotically stable if small perturbations decay to zero in time. When the parameter $\lambda$ is varied one solution may persist but become unstable as $\lambda$ crosses a critical value $\lambda_{c}$, and it is at such a transition point that new solutions may bifurcate from the known solution. In Figure 1 unstable solutions are represented by dashed lines.

A very simple nonlinear partial differential equation which exhibits the

Received by the editors December 15, 1979.

1980 Mathematics Subject Classification. Primary 35B32, 35B35; Secondary 20C99, 76E15.

'The preparation of this paper was supported by NSF Grant MCS 78-00415. 
transition phenomena depicted in Figure 1(a) is

$$
\frac{\partial u}{\partial t}=\Delta u+\lambda u+u^{3},\left.\quad u\right|_{\partial D}=0
$$

where $D$ is a smoothly bounded domain in $\mathbf{R}^{n}$. The equilibrium states are given by solutions of the time independent equations (set $\partial u / \partial t=0$ ). One solution valid for all $\lambda$ is clearly $u=0$; this solution becomes unstable at $\lambda=\lambda_{1}$, the first eigenvalue of the Laplacian on $D: \Delta \varphi_{1}+\lambda_{1} \varphi_{1}=0,\left.\varphi_{1}\right|_{\partial D}=$ 0 . For $\lambda>\lambda_{1}$ there are (at least) three solutions of the nonlinear equilibrium equation. The structure of the solution set in the vicinity of $\left(\lambda_{1}, 0\right)$ is given in Figure 1(a); the new bifurcating solutions are stable.

The Laplacian has a series of eigenvalues $\lambda_{1}<\lambda_{2}<\ldots$ tending to infinity, and all of these eigenvalues are potential bifurcation points. The investigation of the branch points $\left(\lambda_{j}, 0\right)$ for $j>1$ is, however, largely academic, since all of these later solutions must be unstable.

The area of fluid mechanics is a rich source of instability and bifurcation phenomena and the subject has always stimulated the development of mathematical analysis. It has the advantage that accurate, tractable mathematical models are known (for example, the Navier-Stokes equations), for which many careful experimental studies have been made.

One problem in fluid mechanics which has attracted much interest in recent years is the so-called Bénard problem, named after $\mathbf{H}$. Bénard who first performed his series of experiments at the turn of the century. In these experiments a layer of fluid is heated from below, causing an instability to develop in the fluid layer when the temperature drop across the layer exceeds a certain critical value. The resulting instability takes the form of convective motions in the fluid. One of the most striking features of the experiment is the regular cellular, in fact crystallographic structure exhibited by these fluid motions.

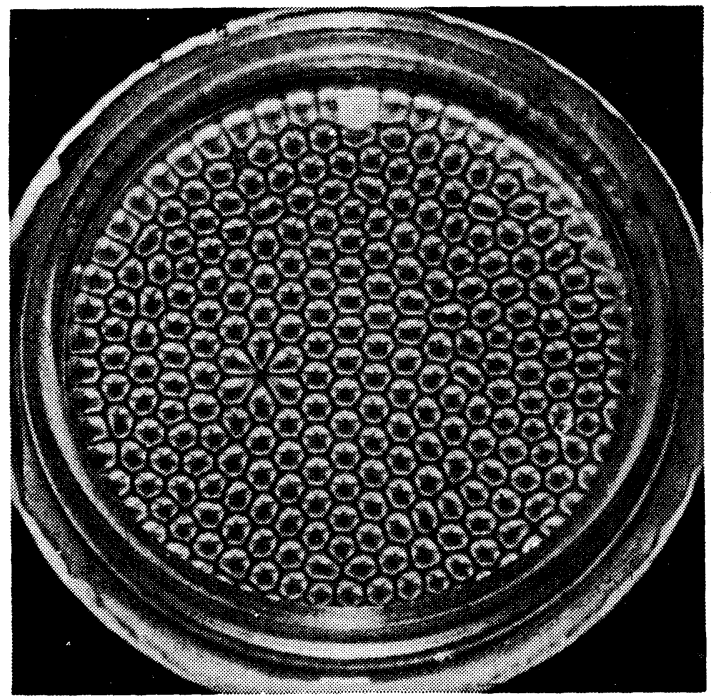

FIGURE 2

Hexagonal Cells in the Bénard problem; E. L. Koschmieder [49] 
The formation of convection cells in the Bénard problem furnishes an excellent example of what is called a "symmetry breaking instability". Prior to the onset of instability the solution is invariant under the entire group of rigid motions (in the idealized infinite plane layer model; see §III.4) whereas the bifurcating convective motions are invariant only under a crystallographic subgroup. Symmetry is broken "spontaneously", because the symmetry group of the equations is unchanged, while the bifurcating solutions have a smaller symmetry group.

The appearance of the Bénard cells is not an isolated example, but rather typifies a broad range of phenomena in nature. Bifurcation, and symmetry breaking instabilities in particular, plays an important role in a diversity of physical disciplines, including buckling problems in elasticity, pattern formation in reaction-diffusion problems, convective flows in geophysical phenomena, neurobiology, physical chemistry, and statistical physics, to name a few. The formation of order in dissipative structures is a subject currently of interest not only to physicists, but also to biologists endeavoring to explain the mechanisms of morphogenesis and pattern formation in evolving biological organisms. The thesis that order-disorder transitions in biological structures can be modeled by systems of partial differential equations describing the processes of reaction and diffusion was advanced by A. M. Turing in 1952 [96] and is today the basis of much of the work in theoretical biology and morphogenesis. (See Fife [19].)

I should like to present, in this article, a summary of the principal mathematical techniques of bifurcation theory, an account of some of the physical problems in which bifurcation and symmetry breaking play an important role, and some open mathematical problems whose resolution I believe is germane to future significant progress. The principal tool for dealing with symmetry breaking bifurcations is group representation theory; this aspect of the subject will be discussed in detail in Chapter III.

\section{Basic techniques of bifurcation theory.}

1. Nonlinear functional calculus. A nonlinear mapping $F$ from a Banach space $\mathcal{E}$ to $\mathcal{F}$ is said to be Fréchet differentiable at a point $u$ provided there is a bounded linear operator $A$ from $\mathcal{E}$ to $\mathscr{F}$ such that the quantity $R(u ; h)=F(u+h)-F(u)-A h$ is $o(h)$ as $h \rightarrow 0$; that is

$$
\lim _{\|h\| \rightarrow 0} \frac{\|R(u ; h)\|}{\|h\|}=0 .
$$

We denote the Fréchet derivative of $F$ at $u$ by $F^{\prime}(u)$ or by $F_{u}$; when it exists it may be computed by the usual formula

$$
F^{\prime}(u) h=\lim _{t \rightarrow 0} \frac{F(u+t h)-F(u)}{t} .
$$

The functional calculus familiar in the theory of finite-dimensional mappings from $\mathbf{R}^{n}$ to $\mathbf{R}^{m}$ carries over directly to the infinite-dimensional case with no difficulty. The chain rule, Taylor's theorem, and the implicit function theorem in a Banach space are all valid, and are extremely useful in nonlinear 
analysis. The following version of the implicit function theorem is adequate for most applications:

THeOREM 1: IMPLICIT FUNCTION THEOREM. Let $\Lambda, \mathcal{E}, \mathcal{F}$ be Banach spaces and let $G$ be a Fréchet differentiable mapping from a domain $U \subset \Lambda \times \mathcal{E}$ to $\mathscr{F}$. Suppose $G\left(\lambda_{0}, u_{0}\right)=0$ and $G_{u}\left(\lambda_{0}, u_{0}\right)$ is an isomorphism from $\mathcal{E}$ to $\mathscr{F}$. Then locally, for $\left|\lambda-\lambda_{0}\right|$ sufficiently small, there is a differentiable mapping $u(\lambda)$ from $\Lambda$ to $\mathcal{E}$, with $(\lambda, u(\lambda)) \subset U$, such that $G(\lambda, u(\lambda))=0$. Furthermore, in a sufficiently small neighborhood $U^{\prime} \subset U,(\lambda, u(\lambda))$ is the only solution of $G=0$. If $G$ is $C^{k}$ then $u$ is $C^{k}$. If $\Lambda, \mathcal{E}$, and $\mathscr{F}$ are complex Banach spaces and $G$ is Fréchet differentiable, then $G$ is analytic and $u$ is analytic in $\lambda$.

This theorem is proved by a contraction mapping argument. See Dieudonné [16] and also [82] for some details of the analytic case in infinite dimensions.

From Theorem 1 it follows that if $G$ vanishes at $\left(\lambda_{0}, u_{0}\right)$ and $G_{u}$ is invertible there, then there is locally a smooth curve of solutions $u(\lambda)$ through $\left(\lambda_{0}, u_{0}\right)$. Bifurcation may occur, however, when $G_{u}\left(\lambda_{0}, u_{0}\right)$ is not invertible. In this article we restrict ourselves to problems in which $G_{u}$ is a Fredholm operator of index zero-that is, $G_{u}$ has a closed range $\mathscr{R} \subset \mathscr{F}$ and finite-dimensional kernel $\Re$, with $\operatorname{dim} \Re=\operatorname{codim} \Re$. The assumption that $G_{u}$ is a Fredholm operator is satisfied in many applied problems where $G$ is typically an elliptic system or a completely continuous integral operator. In some cases, for example when working on unbounded domains, it is the choice of function space which determines whether $G_{u}$ is Fredholm or not; for example, by imposing some kind of periodicity conditions it may be possible to ensure that $G_{u}$ be Fredholm.

2. Principle of linearized stability. Suppose now that the dynamics of a physical system are governed by the evolution equations

$$
\frac{\partial u}{\partial t}=G(\lambda, u)
$$

Let $U(t ; f)$ denote the solution of this equation with initial data $U(0 ; f)=f$, and suppose that $u_{0}=u_{0}(\lambda)$ is an equilibrium solution, that is, $G\left(\lambda, u_{0}(\lambda)\right)=$ 0 . The equilibrium $u_{0}$ is stable if given any $t>0$ there is a $\delta>0$ such that $\left\|u(t ; f)-u_{0}\right\|<t$ for all $t>0$ whenever $\left\|f-u_{0}\right\|<\delta$. Furthermore, $u_{0}$ is asymptotically stable if in addition $u(t) \rightarrow u_{0}$ as $t \rightarrow \infty$. If (2) is a system of ordinary differential equations, that is, if the Banach space $\mathcal{E}$ is finite dimensional, then Lyapounov's first theorem states that $u_{0}$ is asymptotically stable if all eigenvalues of the $G_{u}\left(\lambda, u_{0}\right)$ have negative real parts, and $u_{0}$ is unstable if some eigenvalues of $G_{u}\left(\lambda, u_{0}\right)$ have positive real parts.

Lyapounov's theorem has been extended to dissipative systems of nonlinear partial differential equations; for example, to parabolic systems and to the Navier-Stokes equations, which govern the dynamics of a viscous incompressible fluid [25], [35], [43], [47], [68], [79]. By the principle of linearized stability we mean that the stability of an equilibrium solution $u_{0}$ is determined formally by the spectrum of the linearized operator $G_{u}\left(\lambda, u_{0}\right)$. The phrase "linearized stability" refers to the fact that the full nonlinear equations for the perturbations are replaced by the linearized equations $v_{t}=G_{u}\left(\lambda, u_{0}\right) v$, and it 
is assumed that the stability of these linearized equations determines that for the full nonlinear equations. This principle is generally accepted as valid in the applied literature, and stability is determined formally by solving the linear eigenvalue problem $G_{u}\left(\lambda, u_{0}\right) \varphi=\sigma \varphi$.

Now suppose we have a known solution $u(\lambda)$ of the equilibrium equations $G(\lambda, u(\lambda))=0$ and let $L(\lambda)=G_{u}(\lambda, u(\lambda))$. Suppose that as $\lambda$ crosses $\lambda_{0}$ one or more eigenvalues of $L(\lambda)$ cross the imaginary axis from the left to the right-half plane. This is precisely the situation when $u(\lambda)$ becomes unstable. Then $L\left(\lambda_{0}\right)$ has eigenvalues on the imaginary axis. If some of these eigenvalues lie on the origin, then $\left(\lambda_{0}, u\left(\lambda_{0}\right)\right)$ is a possible bifurcation point, for in this case the implicit function theorem can no longer be invoked to guarantee the existence of a unique solution curve through $\left(\lambda_{0}, u\left(\lambda_{0}\right)\right)$.

3. Bifurcation at a simple eigenvalue. When $u(\lambda)$ loses stability by virtue of a simple eigenvalue crossing the origin a fairly general result is available.

THEOREM 2. Let $G(\lambda, u)$ be an analytic mapping and suppose there is a known solution $u(\lambda)$ of (1) which becomes unstable by virtue of a simple isolated eigenvalue $\sigma(\lambda)$ of $G_{u}(\lambda, u)$ crossing the origin as $\lambda$ crosses $\lambda_{0}$, and let $G_{u}$ be a Fredholm operator. Assume $\sigma\left(\lambda_{0}\right)=0$ and $\sigma^{\prime}\left(\lambda_{0}\right)>0$. Then there is a smooth solution curve $(\lambda(\varepsilon), u(\varepsilon))$ bifurcating from the given solution $u(\lambda)$ at $\left(\lambda_{0}, u\left(\lambda_{0}\right)\right)$. The bifurcating solutions are stable when they appear supercritically $\left(\lambda>\lambda_{0}\right)$ and unstable subcritically $\left(\lambda<\lambda_{0}\right)$.

The three possible situations are depicted below in Figure 3.

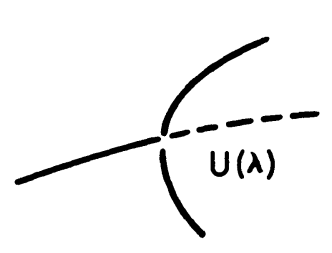

(a)

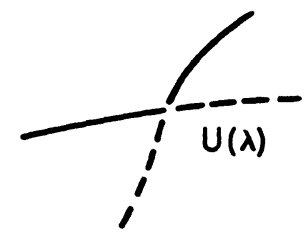

(b)

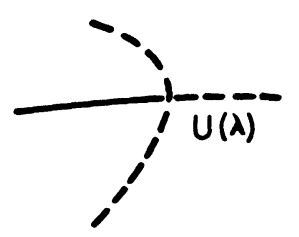

(c)

Figure 3.

Bifurcation at a simple eigenvalue, dashed lines denote unstable branches.

Theorem 2 was first stated for ordinary differential equations by $E$. Hopf in his 1942 article [28], but with certain restrictive assumptions on $\lambda(\varepsilon)$, namely that $\lambda^{\prime}(0)$ and $\lambda^{\prime \prime}(0)$ are not both zero. The indicated method was to compute the perturbation series for the critical eigenvalue along the bifurcating branch. The general infinite-dimensional result, without the restrictive assumptions on $\lambda(\varepsilon)$, was proved using a topological degree argument [80]. (See also [14] for a perturbative proof.) $\mathrm{H}$. Weinberger [100] has generalized these results to the case where $\sigma^{\prime}\left(\lambda_{0}\right)=0$. In that case the bifurcation consists in general of several smooth curves intersecting in a point, with the stability of each branch alternating as one goes consecutively from branch to branch. Stability results based on topological degree arguments have also been obtained by T. B. Benjamin [4]. 
4. Bifurcation of periodic solutions. If $u(\lambda)$ loses stability by virtue of a pair of complex conjugate eigenvalues crossing the imaginary axis then, under suitable but reasonable technical conditions, one may prove the existence of bifurcating time periodic solutions of the evolution equation $u_{t}=G(\lambda, u)$. Let $L(\lambda)=G_{u}(\lambda, u(\lambda))$ and suppose $\gamma(\lambda)$ is the critical eigenvalue of $L(\lambda)$; thus $L(\lambda) \varphi(\lambda)=\gamma(\lambda) \varphi(\lambda)$. For real equations (that is, if $G$ preserves the space of $\mathscr{E}$ of real functions) $L(\lambda)$ computes with complex conjugation, so $\bar{\gamma}(\lambda)$ is also an eigenvalue with eigenvector $\bar{\varphi}(\lambda)$. We have

THEOREM 3. Let $G(\lambda, u)$ be real analytic and suppose that $L(\lambda)$ generates an analytic semigroup. Suppose furthermore that $\gamma\left(\lambda_{c}\right)=i \omega_{0}, \operatorname{Re} \gamma^{\prime}\left(\lambda_{c}\right)>0$, and ni $\omega_{0}$ is not in the spectrum of $L\left(\lambda_{c}\right)$ for $n=2,3, \ldots$. Then there exists $a$ one-parameter analytic family $(\lambda(\varepsilon), \omega(\varepsilon), u(s, \varepsilon))$ such that

(i) $\lambda(0)=\lambda_{c}, \omega(0)=\omega_{0}, u(s, 0)=u\left(\lambda_{c}\right)$,

(ii) $u(s, \varepsilon)$ is $2 \pi$-periodic in $s$,

(iii) $\lambda(\varepsilon), u(\omega(\varepsilon) t, \varepsilon)$ is a solution of the time dependent equations.

This bifurcating time periodic family is unique up to phase shifts and occurs on one side of criticality only. If $\lambda^{\prime \prime}(0) \neq 0$, the branching solutions are stable if they appear supercritically and unstable if they appear subcritically.

E. Hopf first proved the result in this generality for the finite-dimensional case in 1942 [28] and also discussed the possibility of bifurcation of time periodic solutions for the Navier-Stokes equations. The first proofs of the theorem for the Navier-Stokes equations were given by Iudovic [38], Iooss [31], and myself [81], and an improved version was subsequently obtained by D. Joseph and myself [42]. A careful treatment of the problem, under fairly general hypotheses, has been given in a recent paper by Crandall and Rabinowitz [15]. The phenomenon of time periodic oscillations is important not only in fluid mechanics, but also in any dynamical system; for example in reaction-diffusion problems in chemical kinetics, in biological and ecological systems, and lasers [23], [26] to name a few. An alternative proof of the Hopf bifurcation theory, based on the center manifold theorem in a Banach space, has been developed by Marsden and McCracken [58].

The stability statements in Theorem 3 refer to the behavior of the Floquet exponents as one continues along the bifurcating solutions. Rigorous proofs that the Floquet exponents determine the stability for the nonlinear equations have been given by Iooss [31] and Iudovich [37].

5. Hysteresis. This section must necessarily take on somewhat of a metaphysical flavor, for I want to discuss a phenomenon which is quite common in applications, but, because it pertains to the structure of the solution set in the large, is often beyond the reach of rigorous mathematical treatment. Consider the diagram in Figure 4. Again, stable solutions are indicated by solid lines and unstable solutions by dashed lines. According to Theorem 2 subcritical solutions bifurcating at a simple eigenvalue are unstable and supercritical solutions are stable. At multiple eigenvalues, however, this need no longer be the case, and a transcritical branch may be unstable on both sides of criticality. Suppose, however, that the subcritical branch has one unstable mode, and that this subcritical branch "bends back" as shown in the figure. Precisely at the point $\left(\lambda_{0}, u_{0}\right), G_{u}$ must have a nontrivial null space. 
For, let $(\lambda(\varepsilon), u(\varepsilon))$ be a regular parametrization of the solution curve. Then at $\left(\lambda_{0}, u_{0}\right), d \lambda / d \varepsilon=0$ and $d u / d \varepsilon \neq 0$, and differentiating $G(\lambda(\varepsilon), u(\varepsilon))$ along the curve one obtains that $G_{u} u^{\prime}=0$. If $\operatorname{dim} \operatorname{ker} G_{u}=1$ then it can be shown that an eigenvalue of $G_{u}(\lambda(\varepsilon), u(\varepsilon))$ crosses the origin as we pass through $\left(\lambda_{0}, u_{0}\right)$. If the eigenvalue which crosses is the unstable one associated with the lower branch, then the solution will regain stability as we move to the upper branch. If the solution structure is as in Figure 4 then the following effects would be observed. As $\lambda$ is increased past $\lambda_{c}$ the basic solution becomes unstable and the system makes a rapid transition to the new, nontrivial solution, as indicated by the vertical line $(a)$; as $\lambda$ is decreased the system moves along $(b)$ until the point $\lambda_{0}$ is reached, when it drops back to the basic state $(c)$. This behavior is detected in many physical systems, and in fact the nontrivial branch sometimes extends very deeply into the subcritical region. The effect associated with Figure 4 may be called hysteresis in analogy with similar effects observed in ferromagnets, namely the transition path from state $A$ to $B$ is different than that from $B$ to $A$.

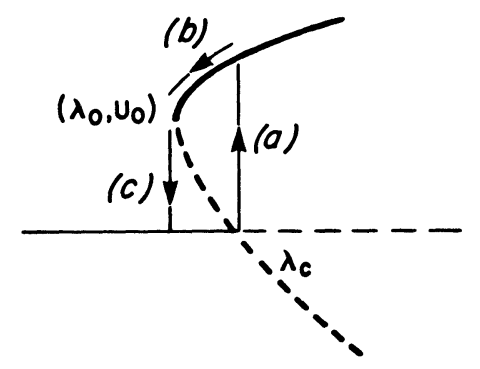

FIGURE 4

6. Lyapounov-Schmidt procedure. We now discuss a general method for reducing an infinite-dimensional bifurcation problem to a finite-dimensional, algebraic one. We assume that $L(\lambda)=G_{u}(\lambda, u(\lambda))$ has, at $\lambda=\lambda_{c}$, an $n$-fold eigenvalue at the origin, that is, that $\operatorname{dim} \operatorname{ker} L\left(\lambda_{c}\right)=n$. For simplicity, let us assume the origin transformed so that $\lambda_{c}=0$ and $u\left(\lambda_{c}\right)=0$, and write $L_{0}=G_{u}(0,0)$. It is very natural to make the assumption in applications that $\mathcal{E} \subseteq \mathscr{F}$ (for example if $G$ is a second-order elliptic operator then typically $\mathcal{E}=C_{2, \alpha}$ and $\mathscr{F}=C_{\alpha}$, and this assumption somewhat simplifies the arguments. Let $\Re=\operatorname{ker} L_{0}=\left[\varphi_{1}, \ldots, \varphi_{n}\right]$ and let $P$ be the projection onto this linear space which commutes with $L_{0}$. Then $P$ must take the form

$$
P u=\sum_{j=1}^{n}\left\langle u, \varphi_{j}^{*}\right\rangle \varphi_{j}
$$

where the $\varphi_{j}^{*} \in \mathcal{F}^{*} \subset \mathcal{E}^{*}$ are null functions of the adjoint operator $L_{0}^{*}$ and $\left\langle\varphi_{i}, \varphi_{j}^{*}\right\rangle=\delta_{i j} . P$ is a linear operator from $\mathscr{F}$ to $\mathscr{F}$, hence can be regarded as a mapping from $\mathcal{E}$ to itself as well, and $Q=I-P$ is a projection onto the range of $L_{0}$ in $\mathcal{F}$. Using $P$ and $Q$ the equation $G(\lambda, u)=0$ can be decomposed into the system of equations

$$
Q G(\lambda, v+\psi)=0, \quad P G(\lambda, v+\psi)=0
$$

where $v=P u$ and $\psi=Q u$. The first equation is solved for $\psi=\psi(\lambda, v)$ by 
applying the implicit function theorem, and then this result is substituted into the second equation, thus obtaining the bifurcation equations

$$
F(\lambda, v) \equiv P G(\lambda, v+\psi(\lambda, v))=0 .
$$

Solutions of the bifurcation equation are in one-to-one correspondence with solutions of the original system sufficiently close to the bifurcation point.

The procedure we have just described is known as the Lyapounov-Schmidt method; the solution of the infinite-dimensional problem is reduced to an algebraic problem. In addition to finding all solutions of the bifurcation equations we shall want to determine their stability properties. A second method for reducing an infinite-dimensional problem to a finite one at the critical point is by way of the center manifold theorem. This method will be described in $\$ 8$.

7. Reduced bifurcation equations. Having derived the bifurcation mapping $F$, let us expand this mapping in a power series in $v$ :

$$
F(\lambda, v)=A(\lambda) v+B_{2}(\lambda ; v, v)+B_{3}(\lambda ; v, v, v)+\cdots
$$

where $A(\lambda)$ is a linear mapping from $\mathscr{N}$ to $\mathscr{T}, B_{2}$ is a quadratic mapping, and so forth. By scaling the variables $\lambda$ and $v$ as follows

$$
v=\varepsilon^{\alpha} \xi, \quad \lambda=\varepsilon^{\beta} \tau
$$

with an appropriate choice of the exponents $\alpha$ and $\beta F$ takes the form

$$
F(\lambda, v)=F\left(\varepsilon^{\beta} \tau, \varepsilon^{\alpha} \xi\right)=\varepsilon^{\gamma} Q(\xi, \tau)+\varepsilon^{\gamma+1} Q_{1}(\xi, \tau, \varepsilon) .
$$

Dividing by $\varepsilon^{\gamma}$ and letting $\gamma \rightarrow 0$ we arrive at the reduced bifurcation equations $Q(\xi, \tau)=0$. The choice of the exponents $\alpha$ and $\beta$ can be determined from a Newton diagram analysis of $F$ [77], [83], [98]. One would like to obtain as much information as possible from an analysis of the reduced bifurcation equations (3). If $\left(\xi_{0}, \tau_{0}\right)$ is a solution of the reduced bifurcation equations and $Q_{\xi}\left(\xi_{0}, \tau_{0}\right)$ is invertible, then a solution to the full equations can be obtained by an application of the implicit function theorem. In fact, this solution takes the form

$$
v=\varepsilon^{\alpha}\left(\xi_{0}+\varepsilon, \xi_{1}+\cdots\right), \quad \tau=\varepsilon^{\beta} \tau_{0} .
$$

On the other hand, in applications where the problem is invariant under the action of a continuous symmetry group, $Q_{\xi}$ will in general be singular, and the standard form of the implicit function theorem can no longer be applied. In particular cases (e.g. the Bénard problem [83]) solutions of the reduced bifurcation equations can be extended to solutions of the full equations, even in the presence of a continuous transformation group, but the general case is an open problem.

8. Stability of bifurcating solutions. The stability of the bifurcating solutions can sometimes be determined, in the neighborhood of the branch point, by computing the eigenvalues of the Jacobian $Q_{\xi}\left(\tau_{0}, \xi_{0}\right)$. If $(\lambda(\varepsilon), u(\varepsilon))$ is the bifurcating branch let $L(\varepsilon)=G_{u}(\lambda(\varepsilon), u(\varepsilon))$. $L(0)$ has an $n$-fold eigenvalue at the origin, and, according to standard perturbation theory for an eigenvalue of finite multiplicity, the spectrum of $L(\varepsilon)$ in the neigborhood of the origin is given by the eigenvalues of an $n \times n$ matrix $B(\varepsilon)$. 
THEOREM 4 [84]. $B(\varepsilon)=\varepsilon^{\gamma-\alpha} Q_{\xi}\left(\tau_{0}, \xi_{0}\right)+O\left(\varepsilon^{\gamma-\alpha+1}\right)$ where $v$ and $\tau$ are scaled as in (4).

In particular, if $F(\lambda, v)=\lambda A v+B_{k}(v)+O\left(|\lambda|^{2}+\|v\|^{k+1}\right)$ then $\alpha+\beta=$ $\gamma$ and $B(\varepsilon)=\varepsilon^{\beta} Q_{\xi}\left(\tau_{0}, \xi_{0}\right)+O\left(\varepsilon^{\beta+1}\right)$. If $\beta$ is even, bifurcation is one sided and supercritical (subcritical) solutions are obtained by solving the reduced bifurcation equations for $\tau_{0}=1\left(\tau_{0}=-1\right)$. When $\beta$ is odd the bifurcation is transcritical and we may take $\tau_{0}=1$.

COROLlaRY 5 [77], [86]. When $F_{\lambda v}=A$ is nonsingular

$$
B(\varepsilon)=\varepsilon^{\beta} Q_{\xi}\left(\tau_{0}, \xi_{0}\right)+O\left(\varepsilon^{\beta+1}\right) .
$$

For even $\beta$ unstable modes of the branching solutions are in one-to-one correspondence with the positive eigenvalues of $Q_{\xi}$. For odd $\beta$ bifurcation is transcritical and unstable modes of the supercritical (subcritical) branch are in one-to-one correspondence with the positive (negative) eigenvalues of $Q_{\xi}$.

9. Center manifold theorem. The center manifold theorem in a Banach space is an alternative method for reducing an infinite-dimensional problem to a finite-dimensional one. The theorem in the case of smooth diffeomorphisms was given by $\mathrm{O}$. E. Lanford [52].

Theorem 6. Center MANifold THEOREM IN A BANACH SPACE. Let $Z$ be $a$ Banach space and let $\psi \in C^{k+1}(Z)$, with $\psi(0)=0$. Let $\psi^{\prime}(0)$ have spectral radius 1 and let the spectrum of $\psi^{\prime}(0)$ be the union of a part on the unit circle and a part inside. (This is the case if $\psi^{\prime}(0)$ is compact.) Let $Y$ denote the generalized eigenspace of $\psi^{\prime}(0)$ belonging to the spectrum of $\psi^{\prime}(0)$ on the unit circle, and assume $\operatorname{dim} Y=d<+\infty$. Then there exists a neighborhood $U$ of 0 in $Z$ and $a C^{k}$ submanifold $M$ of $U$ of $\operatorname{dim} d$, passing through 0 and tangent to $Y$ at 0 such that

(a) $\psi(M \cap U) \subset M$,

(b) if $\psi^{n}(x) \in U$ for $n=1,2, \ldots$ then, as $n \rightarrow \infty$, dist $\left\|\psi^{n}(x)-M\right\| \rightarrow 0$. $M$ is called a center manifold; in general it is not unique.

The reduction of the infinite case to the finite-dimensional case makes use of a special trick: the center manifold theorem is applied not to $\phi(x, \mu)$ but to

$$
\psi(x, \mu)=(\phi(x, \mu), \mu) .
$$

If $\phi^{\prime}(0,0)$ has two simple complex conjugate eigenvalues of $|z|=1$ then

$$
D \psi=\left(\begin{array}{cc}
\phi_{x} & \phi_{\mu} \\
0 & 1
\end{array}\right)
$$

has a three-dimensional space $Y$ with spectrum on the unit circle. So by the center manifold theorem there is a three-dimensional manifold $M$ in $x-\mu$ space; fixing $\mu$ we obtain a two-dimensional section $M_{\mu}$ which is locally invariant and attracting for $\Phi(0, \mu)$. Now restrict $\Phi(0, \mu)$ to $M_{\mu}$ and we have reduced the situation to a two-dimensional problem.

10. Repeated branching, strange attractors, and turbulence. We have seen that when an equilibrium solution loses stability it may bifurcate to a time-periodic solution. What happens when, at a later stage, that time periodic solution loses stability? The situation is best described in terms of the 
Poincaré map, which is constructed as follows. Take a small section $\mathcal{S}$ of a hyperplane of codimension 1 which intersects a periodic orbit $\gamma$ in a single point $x_{0}$. For a point $x$ in that section let $\phi(x)$ be the point where the trajectory through $x$ next intersects $\mathcal{S}$ going in the same direction. $\phi$ is thus defined on some subset $\mathcal{S}^{\prime}$ of $\mathcal{S}$ and fixes $x_{0}$. Periodic orbits near $\gamma$ show up as fixed points of $\phi$, while subharmonic solutions of period roughly $N \times$ (per $\gamma$ ) correspond to fixed points of $\phi^{(n)}$, the $n$th iterate of $\phi$. The stability of a fixed point $x$ is determined by the spectrum of the Fréchet derivative $\phi(x)$ : $x$ is stable if the spectrum of $\phi^{\prime}$ is contained in the disk $|\sigma|<1$ and unstable if the spectrum of $\phi^{\prime}$ contains points in $|\sigma|>1$. The existence and regularity of the mapping $\phi$ is a consequence of the smooth dependence of the solution on the initial data. This includes the infinite-dimensional case when the differential equations are parabolic and the data is smoothed as time goes forward.

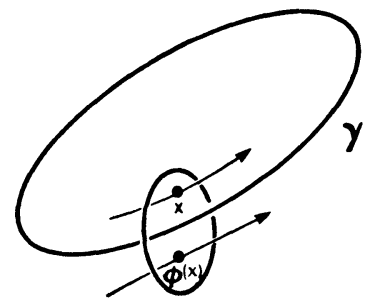

FIGURE 5.

Now let us suppose that as $\mu$ crosses zero the fixed point $x_{0}$ of $\phi$ loses stability by a simple eigenvalue of $\phi_{x}^{\prime}\left(x_{0}, \mu\right)$ crossing through \pm 1 or by a complex conjugate pair of simple eigenvalues $\lambda(\mu), \bar{\lambda}(\mu)$ crossing the unit circle. The multidimensional case (even the infinite-dimensional case) can, under reasonable regularity assumptions, be reduced to the finite-dimensional case by the center manifold theorem, and when $\lambda, \bar{\lambda}$ cross the unit circle we have a two-dimensional bifurcation problem with the following result [52], [65], [74], [76].

THEOREM 7. If $\lambda^{m} \neq 1$ for $m=1,2,3,4$ there exists a coordinate transformation after which $\phi: \mathbf{R}^{2} \times \mathbf{R} \rightarrow \mathbf{R}^{2}$ takes the form $\phi(x, \mu)=N(x, \mu)+$ $O\left(|x|^{5}\right)$ where, in polar coordinates,

$$
N(x, \mu):(r, \varphi) \rightarrow\left((1+\mu) r-f_{1}(\mu) r^{3}, \varphi+\theta(\mu)+f_{3}(\mu) r^{2}\right) .
$$

If $f_{1}(0)>0$ there exists, for small $\mu$, an invariant attracting circle of $\phi(\cdot, \mu)$.

REMARK. If $f_{1}(0)<0$ there exists an attracting invariant circle for $\phi^{-1}(\cdot, \mu)$ for $\mu<0$. These invariant circles appear as bifurcating invariant tori for the original dynamical system. This theorem was announced by Neimark but with the condition $\lambda^{m}(0) \neq 0$ for $m=1, \ldots, 4$ replaced by the condition that the origin be a weak attractor for the diffeomorphism $\phi(\cdot, 0)$ at criticality. It was proved by Sacker in 1964 but appeared only as a New York University technical report. It was proved independently by Ruelle and Takens, who also extended it to the infinite-dimensional case using the center manifold theorem. (See also Lanford [52].) The situation in the strong 
resonance cases $\lambda^{m}=1$ for $m=1,2,3,4$ can be dealt with in greater detail as follows.

THEOREM 8 (IOOSS AND JOSEPH [33]). Assuming sufficient regularity for the diffeomorphism $\phi$ and that $\lambda^{m}(0)=1$ for $m=1,2,3$, or 4 , we have

$m=1$ : bifurcating fixed points of $\phi$; supercritical solutions are stable, subcritical solutions are unstable.

$m=2$ : bifurcating fixed points of $\phi \circ \phi$ appearing on one side of criticality only; supercritical solutions are stable, subcritical solutions unstable.

$m=3$ : bifurcating fixed points of $\phi \circ \phi \circ \phi$; unstable on both sides of criticality.

$m=4:$ three possibilities

(i) one transcritical branch of fixed points of period 4; unstable on both sides of criticality,

(ii) two one-parameter branches of fixed points of period 4; both branches are one-sided (that is, appear on one side of criticality only) and at least one must be unstable,

(iii) no bifurcation at all.

For further details and results, see Iooss' book [32].

It is not in general possible to determine the flow on the invariant torus. One possibility is that the solutions are quasi-periodic with two periods and may be represented in the form $f\left(\omega_{1} t, \omega_{2} t\right)$ where $f$ is $2 \pi$ periodic in each variable and $\omega_{1} / \omega_{2}$ is irrational, but this situation is nongeneric. (See the recent paper by Iooss and Joseph [34] for a more detailed discussion of this aspect of the subject.)

Laudau [51] and Hopf [29] proposed that hydrodynamic turbulence was the result of a cascade of repeated bifurcations: a periodic motion to a quasi-periodic motion with two periods, then two to three, etc. Such solutions can indeed be constructed formally [40], but the perturbation series always lead to small divisors. Moreover, it is impossible to do the calculations in general, and it sometimes is the case that the first bifurcation is subcritical and the system passes directly to a turbulent state. Chenciner and Iooss [10] have shown that under rather stringent (indeed nongeneric) conditions an $n$-torus will bifurcate to an $(n+1)$-torus.

Ruelle and Takens, however, state the following proposition: Let $\omega$ be $a$ constant vector field on a torus $T^{k}(k \geqslant 4)$. In every $C^{k-1}$ small neighborhood of $\omega$ there exists an open set of vector fields possessing a strange attractor. This result shows that systems of ordinary differential equations which exhibit a bifurcation of a 4-torus to a higher-dimensional torus are nongeneric. On this basis Ruelle and Takens disputed the Hopf-Landau view of turbulence; such cascades are nongeneric, and a dynamical system may be expected to exhibit, after only a few bifurcations, a transition to a chaotic state exhibiting no quasi-periodic behavior. This time-dependent state, though complex, should nevertheless exhibit a discernible structure in configuration space and a highly stable behavior. Thus, it should be a set invariant under the flow and which is approached asymptotically as $t \rightarrow \infty$ by all data initially sufficiently close. Furthermore it should be neither an equilibrium solution nor a periodic one. Such stable, aperiodic attracting sets are called "strange attractors". 
Strange attractors are difficult to deal with analytically and rigorous proofs of their existence for a given dynamical system are rare. (See, however, Levinson [54].) Smale's "horseshoe map" [89] is an example of a diffeomorphism possessing a strange attractor (with diffeomorphisms continuous time is replaced by discrete time, and one looks at the flow under iterates of the mapping). Most of the current work in this area consists of numerical analysis of differential equations or diffeomorphisms. In 1963 E. Lorenz [55], a meteorologist interested in the problem of long-range weather preduction, analyzed numerically the very simple system

$$
\dot{x}=-\sigma x+\sigma y, \quad \dot{y}=-x z+r x-y, \quad \dot{z}=x y-b z .
$$

The solutions of these equations exhibit, for certain values of the parameters $\sigma, r$ and $b$, chaotic behavior and sensitive dependence on initial conditions. The divergence of the vector field is everywhere equal to $-(\sigma+b+1)$ so that volumes in $\mathbf{R}^{3}$ are contracted under time evolution. For

$$
r>\sigma(\sigma+b+3)(\sigma-b-1)^{-1}>0
$$

there are three equilibrium points, none of them stable. Lanford and Ruelle [75] analyzed the solutions by studying a section map associated with the flow. Namely, let $P$ be a point on the plane $z=27$ at which $\dot{z}<0$ and follow the trajectory through $P$ until the orbit again crosses $z=27$ in the downward direction; the new point is denoted by $\phi(P)$. Using a computer with a graphical output Lanford plotted the successive iterates $\phi^{(n)}(P)$. For large $n$ the successive images tend to lie along two point sets $\Gamma$ and $\Gamma^{\prime}$ as in the figure below.

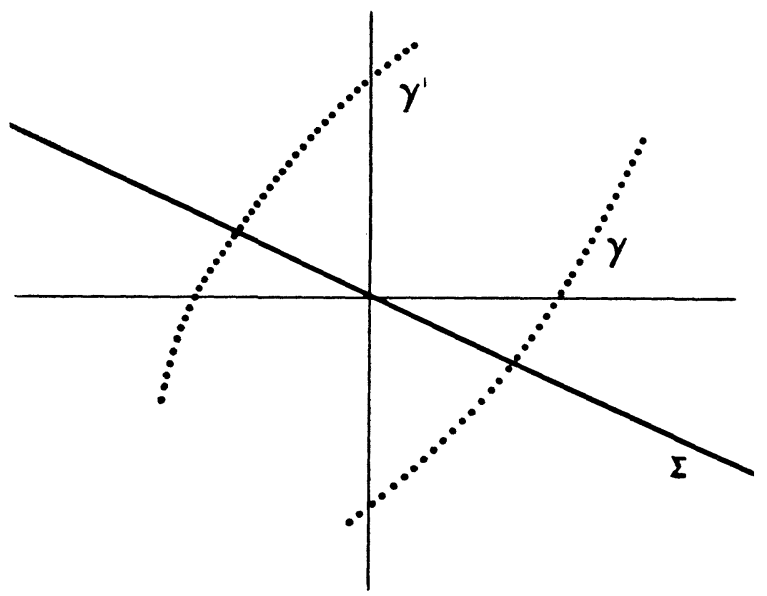

FIGURE 6.

Successive iterates of the section map for the Lorenz equations obtained numerically by Lanford.

(The line $\Sigma$ represents an exceptional set of points which do not come back to cross the plane $z=27$; trajectories through $\Sigma$ tend to the unstable equilibrium point at the origin.) Under successive iterations of $\phi$ points move about $\Gamma$ and $\Gamma^{\prime}$ in an erratic manner. Moreover, two points $P_{1}$ and $P_{2}$ initially close move apart and follow completely different histories under $\phi^{(n)}$; yet 
these sequences nevertheless lie along $\Gamma$ and $\Gamma^{\prime} . \Gamma \cup \Gamma^{\prime}$ represents the intersection of an attracting set $S$ with the plane $z=27$.

Other numerical studies on strange attractors have been carried out by Rössler [73] for simple systems of ordinary differential equations and by Hénon and Pomeau [24] for a simple diffeomorphism of $\mathbf{R}^{2}$. Iterations of nonlinear diffeomorphisms arise directly in models of population dynamics where successive generations are presumed to evolve according to a simple finite difference equation: $x_{n+1}=\phi\left(x_{n}\right)$. See May [59], Guckenheimer [22].

In addition to the computer experiments there are now a number of physical experiments which demonstrate the existence of aperiodic phenomena. Chaotic behavior has been observed in chemical kinetics [88] and has been measured quantitatively in recent experiments with turbulent flow. In 1975 Gollub and Swinney [20] used laser light scattering and photon correlation methods to obtain highly accurate measurements of the power spectrum of the fluid motion in a classical experiment known as the Taylor problem. Such experimental techniques allow one to obtain quantitative information about the frequency spectrum of the flow, and Gollub and Swinney (later with Fenstermacher) compared the results of their experiments with the theoretical discussions of Ruelle and Takens, Joseph and Iooss.

In the Taylor experiment (first performed and analyzed theoretically by $\mathbf{G}$. I. Taylor in 1923 [91]) the region between two concentric cylinders is filled with fluid, and the inner cylinder is rotated. The nondimensional bifurcation parameter is the Reynolds number $R=\Omega_{i} r_{i}\left(r_{0}-r_{i}\right) / \nu$ where $\Omega_{i}$ is the angular velocity of the inner cylinder, $r_{i}$ and $r_{0}$ are the radii of the inner and outer cylinders, and $\nu$ is the kinematic viscosity of the fluid. At small $R$ the flow is purely azimuthal, that is, $v_{r}=v_{z}=0$ and only $v_{\theta}=v_{\theta}(r)$ is nonzero. When $R$ exceeds a critical value $R_{c}$ there is a bifurcation to a stable flow with a horizontal toroidal system of vortices. This is the bifurcation which was studied in 1923 by Taylor. Under a further increase of $R$ the Taylor vortices become unstable to a time-dependent flow, in fact a series of waves moving around the cylinder. These were experimentally studied in detail by Coles [11]. Under a still further increase of $R$ these wavy vortices break down to a more irregular flow.

The power spectra of the radial velocity flow at various values of $R / R_{c}$ are shown in Figure 7

Taylor vortices at $R / R_{c}=1.1$

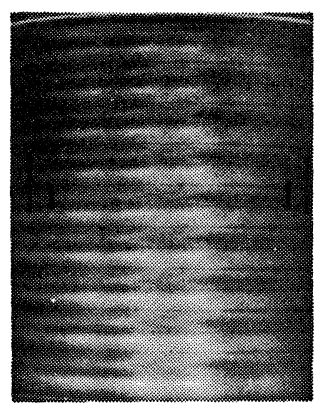




\section{$R / R_{c}=9.6$}

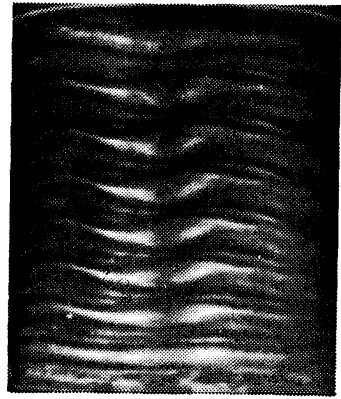

$R / R_{c}=11$

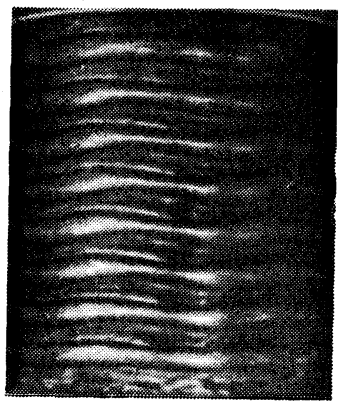

$R / R_{c}=18$

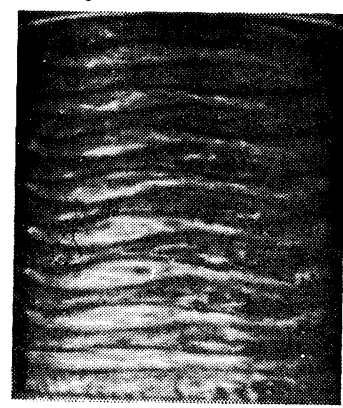

$R / R_{c}=26$

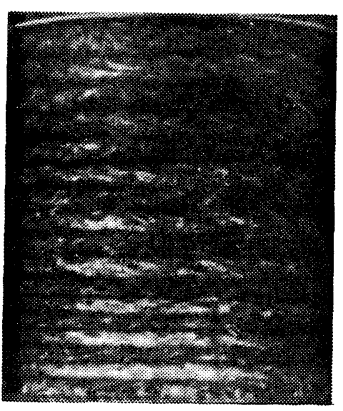

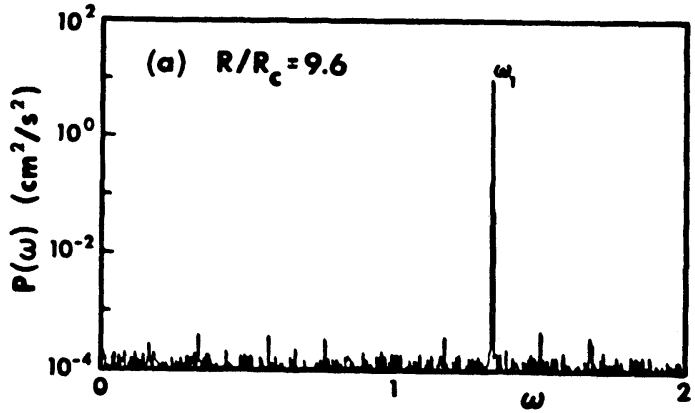
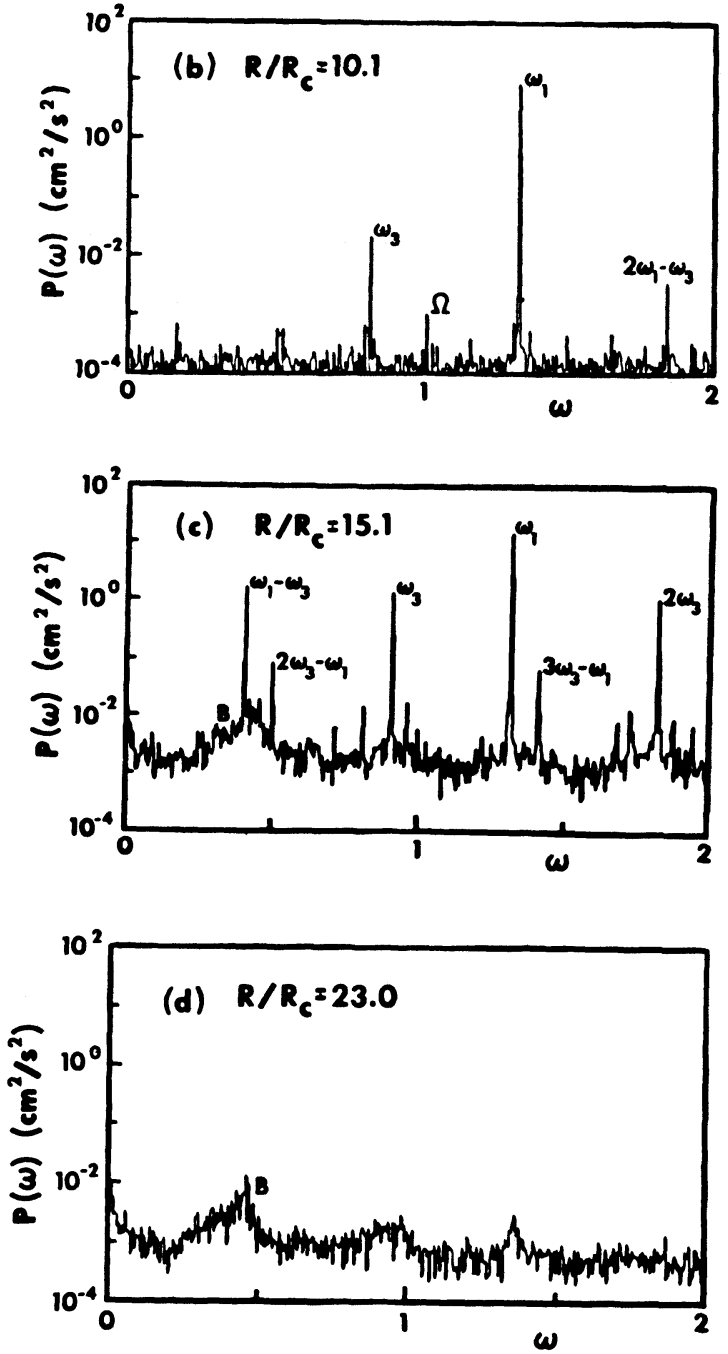

FIGURE 7. Power spectra of the radial velocity of the flow in the Taylor problem obtained in the experiments by Fenstermacher, et al. [17]. 
The time independent Taylor vortices exist in the range $1<R / R_{c}<1.2$. After that the wavy vortices bifurcate and persist stably to about $R / R_{c}=$ $10.1 \pm 0.2$. In this range of $R$ there is a single spike in the power spectrum at $\omega=\omega_{1}$. A second component, denoted by $\omega_{3}$ (a component $\omega_{2}$ found in previous experiments turned out to be a transient), appears at $R / R_{c}=10.1$ \pm 0.2 and persists to $R / R_{c}=19.8 \pm 0.4$, where it disappears. In this range of $\boldsymbol{R}$ the flow is "apparently quasi-periodic, neglecting a weak broadband component" (denoted here by $B$ ).

The onset of the broadband component denoted by $B$ is difficult to determine accurately. It exists with the sharp spikes at $\omega_{1}$ and $\omega_{3}$, and is the only survivor above $R / R_{c}=22.4$. In this range the flow appears to be qualitatively different, "one characterized by a continuous spectrum or, equivalently, a decaying velocity auto correlation function".

These experiments confirm the view that turbulence (1) sets in after only a few bifurcations and (2) cannot be described in terms of a cascade of bifurcations. There are as yet, however, no satisfactory mathematical techniques for giving a theoretical treatment of these observations, even for so simple a system as the Lorenz equations.

11. Imperfections and singularity theory. Bifurcation is, in a sense, an idealized, nongeneric phenomenon; diagrams such as that in Figure 1 are structurally unstable, and small perturbations of the mapping will break the bifurcation diagram. (Consider the behavior of the zero set of $g(x, \lambda, \varepsilon)=\lambda x$ $-x^{3}+\varepsilon$ as $\varepsilon$ varies in a neighborhood of zero.) Since imperfections are present in any physical system we should require that a description of the transition point include the effects of variations in the problem other than the bifurcation parameter. If the idealized bifurcation problem is determined by $g(x, \lambda)=\lambda x-x^{3}=0$, for example, what is the most general form of the solution structure under small perturbations of the system?

Golubitsky and Schaeffer [21] have addressed this question using the theory of singularities of mappings. Let $\mathscr{G}_{n}$ denote the class of germs of all $C^{\infty}$ mappings of $\mathbf{R}^{n} \times \mathbf{R}$ into $\mathbf{R}^{n}$ and associate each element of $\mathscr{B}_{n}$ with a "bifurcation problem". $\mathscr{B}_{n}$ is a module over the ring of $C^{\infty}$ scalar functions with respect to addition and multiplication of functions.

DEFINITION 9. Two bifurcation problems $G, \bar{G} \in \mathscr{B}_{n}$ are contact equivalent if there exists a linear transformation $T_{x, \lambda}$ and orientation-preserving coordinate transformations $X(x, \lambda)$ and $\Lambda(\lambda)$, with $X(0,0)=\Lambda(0)=0$, such that

$$
G(x, \lambda)=T_{x, \lambda} \bar{G}(X(x, \lambda), \Lambda(\lambda)) .
$$

Of course, the analysis is entirely local here.

Let $G \in \mathscr{B}_{n}$ and let $O_{G}$ be the orbit of $G$ under contact equivalence, that is $O_{G}=\{\bar{G} \mid \bar{G}$ is contact equivalent to $G\} . O_{G}$ may be regarded as an infinitedimensional submanifold of $\mathscr{B}_{n}$ whose tangent space $T_{G}$ at $G$ may be calculated formally by differentiating along one-parameter curves in $O_{G}$ through $G$. Thus, differentiating

$$
G_{t}=T(x, \lambda, t) G(X(x, \lambda, t), \Lambda(\lambda, t))
$$

with respect to $t$ and setting $t=0$, where $T(x, \lambda, 0)=I, X(x, \lambda, 0)=x$, and 
$\Lambda(\lambda, 0)=\lambda$, we get

$$
\dot{G}_{0}=\dot{T}(x, \lambda, 0) G(x, \lambda)+\frac{\partial X^{i}}{\partial t} \frac{\partial G}{\partial x^{i}}+\frac{\partial G}{\partial \lambda} \dot{\Lambda}(\lambda, 0)
$$

For example, when $n=1$,

$$
T G=\left\{a(x, \lambda) G+b(x, \lambda) \frac{\partial G}{\partial x}+c(\lambda) \frac{\partial G}{\partial \lambda}\right\},
$$

for arbitrary smooth functions $a(x, \lambda), b(x, \lambda), c(\lambda)$. TG is an ideal generated by $G, G_{x}$, and $G_{\lambda}$ in the ring $\mathscr{B}_{1}$.

DEFINITION 10. The bifurcation problem $G$ has codimension $k$ if there exists a $k$-dimensional subspace $K$ of $\mathscr{B}_{n}$ such that $\mathfrak{B}_{n}=T_{G} \oplus K$.

THEOREM 11. If $G$ has finite codimension $k$ and $K$ is spanned by $g_{1}, \ldots, g_{k}(x, \lambda)$, then

$$
G(x, \lambda, \alpha)=G(x, \lambda)+\sum_{i=1}^{k} \alpha_{i} g_{i}(x, \lambda)
$$

is a universal unfolding of $G$.

This theorem means that any perturbation of $G$ of the form

$$
G\left(x, \lambda, \varepsilon_{1}, \ldots, \varepsilon_{l}\right)=G+\sum_{i=1}^{l} \varepsilon_{i} h_{i}(x, \lambda)
$$

for smooth $h_{1}, \ldots, h_{l}$ is contact equivalent to $F$, and the coefficients $\alpha_{i}$ depend smoothy on the parameters $\varepsilon_{i}$. Therefore any basic bifurcation problem $G$ with finite codimension can be transformed into an explicitly computable canonical form. If we restrict ourselves to real-valued functions (potentials) of codimension at most 4 , we are led to the so-called " 7 elementary catastrophes" of Thom.

COROlLARY 12. Up to contact equivalence, $F(x, \lambda, p, q)=x^{3}-\lambda x+p x^{2}+$ $q$ is a universal unfolding of $x^{3}-\lambda x$.

Corollary 12 is obtained from Theorem 11 by some elementary manipulations with Taylor's series. Since this is an important technique in singularity theory it is worth presenting here. The tangent space is given by

$$
T G=\left\{a\left(x^{3}-\lambda x\right)+b\left(3 x^{2}-\lambda\right)+c(\lambda) x\right\} .
$$

Note that $x^{3}=\frac{x}{2}\left(3 x^{2}-\lambda\right)-\frac{1}{2}\left(x^{3}-\lambda x\right) \in T G$. We must show that $K=$ $\mathscr{B}^{1} / T G=\operatorname{sp}\left\{1, x^{2}\right\}$. Any $C^{\infty}$ function $f(x, \lambda)$ can be expanded as

$$
f(x, \lambda)=f_{0}(\lambda)+f_{1}(\lambda) x+f_{2}(\lambda) x^{2}+f_{3}(\lambda, x) x^{3}
$$

but since $f_{1}(\lambda) x$ and $x^{3}$ belong to $T G$,

$$
f(x, \lambda) \equiv f_{0}(\lambda)+f_{2}(\lambda) x^{2}(\bmod T G) .
$$

Furthermore $\left(3 x^{2}-\lambda\right) \in T G$ so $\lambda \equiv 3 x^{2}(\bmod T G)$; therefore

$$
\begin{aligned}
f_{0}(\lambda) & =f_{0}(0)+\lambda f_{0}^{\prime}(0)+\lambda^{2} h(\lambda) \\
& \equiv P_{0}+P_{1} x^{2}+9 x^{3}(x h(\lambda))(\bmod T G) \\
& \equiv P_{0}+P_{1} x^{2}(\bmod T G) ;
\end{aligned}
$$


and

$$
\begin{aligned}
f_{2}(\lambda) & =f_{2}(0) x^{2}+g(\lambda) x(\lambda x) \\
& =f_{2}(0) x^{2}+\frac{\lambda^{2} g(\lambda)}{3}(\bmod T G) \\
& =Q_{0}+Q_{1} x^{2}(\bmod T G) .
\end{aligned}
$$

Finally, $f_{0}(\lambda)+f_{2}(\lambda) x^{2} \equiv P_{0}+\tilde{P}_{1} x^{2}$ where $P_{0}$ and $\tilde{P}_{1}$ are constants. The behavior of the zero structure of $F$ is sketched in Figure 8.

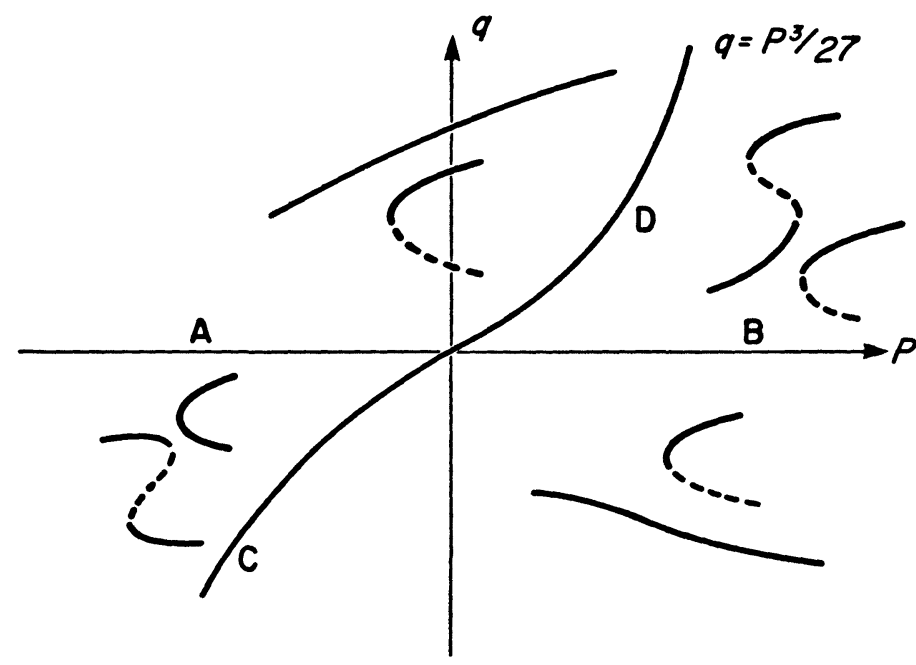

Figure 8.

The structure of the zero set of $F$ changes across the lines $q=0$ and $q=p^{3} / 27$. Real bifurcation can occur only along the exceptional curve $q=0$, hence is nongeneric. Hysteresis sets in upon crossing the curves $C$ and $D$ (Golubitsky and Schaeffer [21]).

The importance of imperfections has long been known among elasticians; see Hunt [30] and Thompson [94]. The effect of imperfections on bifurcation was first studied by J. Keener in his thesis; see Keener [45], Keener and Keller [44], and Reiss [71]. T. B. Benjamin has discussed the effects of imperfection in the bifurcation of the Taylor cells [5].

III. Group representation theory and symmetry breaking.

1. Bifurcation at multiple eigenvalues. Let us now suppose that at criticality, $\operatorname{dim} \operatorname{ker} L_{0}>1$, a situation commonly referred to as "bifurcation at a multiple eigenvalue". The Lyapounov-Schmidt method, described in II.6, is nice in theory, but in practice the computation of even the lowest order terms of the bifurcation equations is extremely complicated. Moreover, systems of $\boldsymbol{n}$ equations in $n$ unknowns can display a complex solution structure and the algebraic problem in general can be quite complex. The results of Theorem 2 concerning the stability of bifurcating solutions are no longer valid at multiple eigenvalues. 
In many problems of physical interest, however, the multiplicity of the branch point arises from an underlying symmetry of the problem. Just as in quantum mechanics, where the invariance of the Hamiltonian under a symmetry group leads to degeneracy of the energy levels, group invariance of a system of nonlinear equations leads to multiple eigenvalue branch points. The physical consequence of this situation is that the bifurcation point is characterized by a symmetry-breaking bifurcation. By applying methods of group representation theory it is possible to compute the structure of the bifurcation equations directly from a knowledge of the transformation group, thus bypassing the complicated Lyapounov-Schmidt procedure. In this way we are able to analyze and classify bifurcation phenomena on geometrical grounds, and thus build a unified mathematical theory of bifurcation at multiple eigenvalues.

2. Covariance of the bifurcation equations. Let $T_{g}$ be a linear representation of a group $\mathcal{G}$ on the Banach spaces $\mathcal{E}$ and $\mathscr{F}$ and assume that $T_{g} G(\lambda, u)=$ $G\left(\lambda, T_{g} u\right)$. This covariance of the mapping $G$ is a natural assumption in physical theories and is a mathematical expression of the axiom that the equations of mathematical physics be independent of the observer. For example, the Navier-Stokes equations

$$
\Delta u_{k}-\frac{\partial p}{\partial x_{k}}=u_{j} \frac{\partial u_{k}}{\partial x_{j}}, \quad \frac{\partial u_{k}}{\partial u_{k}}=0
$$

are covariant with respect to the representation

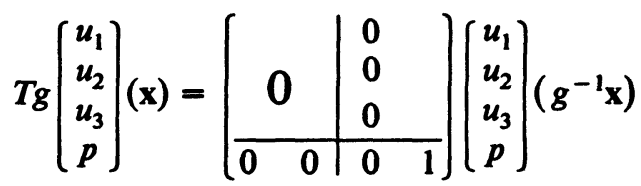

of the group of rigid motions; here $g x=O x+a$ where $a \in R^{3}$ and $O$ is a $3 \times 3$ orthogonal matrix.

Moreover, in mathematical modeling, the introduction of a high degree of symmetry into the geometry simplifies the analysis, so that problems arising in applications are found to have a nontrivial symmetry group.

From covariance it follows that $T_{g} G_{u}(\lambda, u)=G_{u}\left(\lambda, T_{g} u\right) T_{g}$; so if $u_{0}$ is an invariant solution of $\mathcal{G}$ then $T_{g} L_{0}=L_{0} T_{g}$. Therefore $\mathscr{T}_{0}=\operatorname{ker} L_{0}$ is invariant under $T_{g}$. If $\operatorname{dim} \Re_{0}<+\infty$, as is often the case in applications, $\Gamma \equiv T_{g \mid \Re_{0}}$ is a finite-dimensional representation of $\mathcal{G}$. Writing the bifurcation equations in the form $F(\lambda, v)=0$, where $v \in \tau_{0}$ and $F: \mathbf{C} \times \tau_{0} \rightarrow \tau_{0}$, we have the following [83].

THEOREM 13. Let $G(\lambda, u)$ be an analytic operator from a complex Banach space $\mathcal{E}$ to $\mathcal{F}$, covariant with respect to a representation $T_{8}$ of a compact group $\mathcal{G}$. Suppose that $G\left(\lambda_{c}, u_{0}\right)=0, T_{g} u_{0}=u_{0}$ for all $g \in \mathcal{G}$, and $G_{u}\left(\lambda_{c}, u_{0}\right)$ is a Fredholm operator of index zero with kernel $\varkappa_{0}$. Then $\varkappa_{0}$ is invariant under $T_{8}$ and the bifurcation equations $F(\lambda, v)$ are covariant with respect to $\Gamma$, the restriction of $T_{g}$ to $\Re_{0}$; that is $\Gamma_{g} F(\lambda, v)=F\left(\lambda, \Gamma_{g} v\right)$.

This theorem is easily proved by following through the details of the Lyapounov-Schmidt procedure. Each term $B_{k}(\lambda, v, w, \ldots)$ in the expansion 
(3) of $F$ is symmetric in its variables and covariant with respect to the representation $\Gamma_{g}$. In particular, $\Gamma_{g} A(\lambda)=A(\lambda) \Gamma_{g}$; so by Schur's lemma, if $\Re_{0}$ is irreducible $A(\lambda)=\sigma(\lambda) I$, where $I$ is the identity transformation. The higher-order terms $B_{2}, B_{3}, \ldots$ can all be calculated, up to scalar factors, by the methods of group representation theory. For example, if $\mathcal{G}=O(3)$ and $\Re_{0}$ transforms according to the irreducible representation $D^{\prime}$, then the coefficients in the quadratic terms of the bifurcation equation are the ordinary Clebsch-Gordon coefficients for the rotation group. The reduced bifurcation equations are similarly covariant with respect to the representation $\Gamma_{g^{*}}$

In this way, transition phenomena can be categorized according to the geometry of the problem, rather than by the particular physical mechanisms involved. From this point of view, the onset of convection in a spherical geometry, the buckling of a spherical shell, or the onset of ionic currents in a developing spherical egg, can all be given a unified mathematical treatment, even though the physical mechanisms in each of the problems may be vastly different. On the other hand, the specific physical mechanisms in the problem make themselves apparent in the determination of the critical value of $\lambda$ and the transformation properties of the kernel $\varkappa_{0}$. In addition, the physics of the problem determine the values of the scalar parameters multiplying the covariant terms in the bifurcation equations; these parameter values determine the direction of bifurcation (supercritical or subcritical) as well as the stability of the various bifurcating solutions.

Depending on the representation $\Gamma_{g}$, there may be more than one covariant term of lowest degree $k$. In that case we would arrive at a system of reduced equations of the form

$$
\lambda w=a_{1} B_{k}^{(1)}(w)+a_{2} B_{k}^{(2)}(w)+\cdots+a_{l} B_{k}^{(l)}(w)
$$

where the coefficients $a_{1}, \ldots, a_{l}$ are parameters which depend on the original parameters of the systems. The multiplicity $l$ of covariant terms of degree $k$ can be computed directly from a knowledge of the representation $\Gamma$ and does not depend on the particular structure of the equations at hand. In fact, the multiplicity $l$ is precisely the number of times the representation $\Gamma$ is contained in $\left(\Gamma^{\otimes k}\right)_{s}$, where the subscript $s$ denotes the restriction of $\Gamma^{\otimes k}$ to the subspace of completely symmetric tensors. When there are multiple covariant tensors, as in (5), the possibility of selection mechanisms arises. The stability of the various bifurcating solutions depends on the relative sizes of the parameters $a_{1}, \ldots, a_{l}$. The numerical values of the parameters $a_{1}, \ldots, a_{l}$ are determined by the physics of the problem, but in general the computation of their exact dependence on the natural physical parameters of the problem is a difficult matter (equivalent to the direct calculation of the full set of bifurcation equations). Such calculations could be carried out numerically in specific cases, but in order to classify the types of transitions which can take place, it is sufficient to consider the parameters $a_{1}, \ldots, a_{l}$ as free parameters, and investigate the possible transitions which may occur. These matters will be discussed more fully in $\$ 4$.

3. Computational methods. The identification of covariant mappings of a given order with invariant tensors of one higher order gives us, firstly, a way 
to compute the number of independent covariant mappings of a given order based on the group characters. The character of a representation is the trace: $\chi(g)=\operatorname{Tr} T(g)$. If $T(g)$ is a representation the $k$-fold tensor product $T^{\otimes k}(g)$ acting on the space of $k$-linear mappings is defined by $T^{\otimes k}(g) B\left(\mathbf{u}_{1}, \ldots, \mathbf{u}_{k}\right)$ $=B\left(T\left(g^{-1}\right) \mathrm{u}_{1}, \ldots, T\left(g^{-1}\right) \mathrm{u}_{k}\right)$, and the character of this representation is $\chi^{k}$. We always restrict ourselves to the subspace of mappings $B$ which are completely symmetric in their variables, which is invariant under $T^{\otimes k}$. The restriction of the representation $T^{\otimes k}$ to the subspace of symmetric mappings is denoted by $\left(T^{\otimes k}\right)_{s}$ and its character is denoted by $\chi_{(k)}(g)$.

THEOREM 14. Let $T$ be a representation on the vector space $\Re$ of the compact group $\mathcal{G}$ and let $c_{n}(T, \mathcal{G})$ denote the number of completely symmetric n-linear operators on $\Re$ which are covariant with respect to $T$. A generating function for the coefficients is

$$
\sum_{n=0}^{\infty} c_{n}(T, \mathcal{G}) z^{n}=\int_{\mathcal{G}} \operatorname{det}(I-z T(g))^{-1} \bar{\chi}(g) d \mu(g)
$$

where $\chi(g)=\operatorname{Tr} T(g)$ and $\mu(g)$ is the normalized invariant measure on $\mathcal{G}$. (In the case of a finite group, $\mu$ assigns weight $1 /|\mathcal{G}|$ to each element $g \in \mathcal{G}$, where $|\mathcal{G}|$ is the order of $\mathcal{G}$.) Alternatively, the number of $n$-linear covariant mappings may be computed from the formula

$$
\sum_{n=0}^{\infty} c_{n}(T, \mathcal{G}) z^{n}=\int_{\mathcal{G}} \sum_{n=0}^{\infty} \chi_{(n)}(g) \bar{\chi}(g) z^{n} d \mu(g)
$$

where

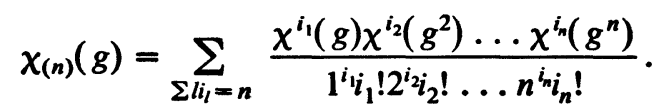

For example, the character $\chi_{(2)}$ is given by

$$
\chi_{(2)}(g)=\frac{1}{2}\left(\chi^{2}(g)+\chi\left(g^{2}\right)\right) \text {. }
$$

This theorem is very closely related to the Molien function which is the generating function for the invariants of a given representation (see Jaric and Birman [39]). The proof of Theorem 14 is given in [86], [87].

EXAMPLE. Let the group in question be $D_{3}$, the symmetry group of the equilateral triangle. This group is generated by the elements $g=\left(\begin{array}{lll}1 & 2 & 3\end{array}\right)$ and $h=\left(\begin{array}{ll}1 & 2\end{array}\right)$. It has three irreducible representations:

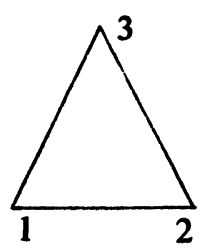

two one-dimensional and one two-dimensional arising from the natural action of $D_{3}$ in the plane. The conjugacy classes are $\{e\},\left\{g, g^{2}\right\}$, and $\left\{h, g h, g^{2} h\right\}$ and the character table is

\begin{tabular}{c|ccc}
$D_{3}$ & $\{e\}$ & $\left\{g, g^{2}\right\}$ & $\left\{h, g h, g^{2} h\right\}$ \\
\hline$\chi^{(1)}$ & 1 & 1 & 1 \\
$\chi^{(2)}$ & 1 & 1 & -1 \\
$\chi^{(3)}$ & 2 & 1 & 0
\end{tabular}


Let $T$ be the representation $T=T^{(1)} \oplus T^{(2)} \oplus T^{(3)}$ and let us compute the number of covariant quadratic mappings. The characters are

\begin{tabular}{c|ccc} 
& $\{e\}$ & $\left\{g, g^{2}\right\}$ & $\left\{h, g h, g^{2} h\right\}$ \\
\hline$\chi$ & 4 & 1 & 0 \\
$\chi_{(2)}$ & 10 & 1 & 2 \\
$\chi_{(2)} \bar{\chi}$ & 40 & 1 & 0
\end{tabular}

Accordingly, the number of maps is

$$
c_{2}\left(T, D_{3}\right)=\frac{1}{6} \sum_{\sigma \in D_{3}} \chi_{(2)}(\sigma) \bar{\chi}(\sigma)=\frac{1}{6}(1 \cdot 40+2 \cdot 1+3 \cdot 0)=7 .
$$

There are thus 7 independent mappings for this representation, and therefore 7 scalar quantities must be tabulated to completely determine the bifurcation. Let us calculate covariant mappings. We represent the vector space $\Re$ as linear polynomials in four variables $x, y, z$, and $\bar{z}$ ( $x$ and $y$ are real). These variables transform as follows under the group action

$$
\begin{array}{ll}
g x=x & h x=x \\
g y=y & h y=-y \\
g z=e^{2 \pi i / 3} z & h z=\bar{z} \\
g \bar{z}=e^{-2 \pi i / 3} \bar{z} & h \bar{z}=z .
\end{array}
$$

(We are using complex conjugate variables $z$ and $\bar{z}$ for the two-dimensional action.) The mapping $F=\left(F_{1}, F_{2}, F_{3}, F_{4}\right)$ must also transform as $x, y, z$, and $\bar{z}$. In particular, $F_{1}$ is an invariant and so is a function of the invariants $x, y^{2},|x|^{2}, z^{3}, \bar{z}^{3}$ :

$$
F_{1}=A\left(x, y^{2},|z|^{2}, z^{3}, \bar{z}^{3}\right) .
$$

$F_{2}$ must transform like $y$, so

$$
F_{2}=y B\left(x, y^{2},|z|^{2}, z^{3}, \bar{z}^{3}\right) .
$$

The functions $z$ and $\bar{z}^{2}$ both transform like $z$, so

$$
F_{3}=z C\left(x, y,|z|^{2}, z^{3}, \bar{z}^{3}\right)+\bar{z}^{2} D\left(x, y,|z|^{2}, z^{3}, \bar{z}^{3}\right) \text {. }
$$

Now applying the group operation $h$, we get

$$
\begin{aligned}
& h F_{3}=F_{4}=F_{3} h, \\
& F_{4}(x, y, z, \bar{z})= F_{3}(x,-y, \bar{z}, z) \\
&= \bar{z} C\left(x,-y,|z|^{2}, \bar{z}^{3},\right)+z^{2} D\left(x,-y,|z|^{2}, \bar{z}^{3}, z^{2}\right) .
\end{aligned}
$$

Now we can write down the lowest order terms, beginning with linear, then quadratic functions of the four variables:

$$
\begin{aligned}
& F_{1}=\lambda_{1} x+a x^{2}+b y^{2}+c|z|^{2}+\cdots \\
& F_{2}=\left(\lambda_{2}+d x\right)+\cdots \\
& F_{3}=\lambda_{3} z+e z y+f x z+g \bar{z}^{2}+\cdots \\
& F_{4}=\lambda_{3} \bar{z}-e \bar{z} y+f x \bar{z}+g z^{2}+\cdots
\end{aligned}
$$


The seven scalars $a, \ldots, g$ index the seven independent quadratic covariant mappings. In practice, seven variables is too many to deal with, and I only give this example as an illustration of the techniques involved. We turn to some computations in actual physical situations.

4. The Bénard problem. The equations modeling convection are the Boussinesq equations

$$
\begin{aligned}
\Delta u_{k}+\delta_{k 3} R \theta-\frac{\partial P}{\partial x_{k}} & =\frac{1}{P_{r}}\left[u_{j} \frac{\partial u_{k}}{\partial x_{j}}+\frac{\partial u_{k}}{\partial t}\right], \\
\Delta \theta+u_{3} & =u_{j} \frac{\partial \theta}{\partial x_{j}}+\frac{\partial \theta}{\partial t}, \\
\frac{\partial u_{j}}{\partial x_{j}} & =0, \\
-\infty & <x_{1}, x_{2}<\infty, \quad 0<x_{3}<1
\end{aligned}
$$

where $P_{r}$ is the Prandtl number, $R$ is the Rayleigh number, and $u_{1}, u_{2}, u_{3}, \theta, P$ are the components of velocity, temperature, and pressure. $R$ is proportional to the temperature drop across the layer, and convection sets in when $R$ exceeds a certain critical value $R_{c}\left(R_{c}=1708\right.$ if both the top and bottom surfaces are rigid). For a more extensive discussion see D. Joseph's book [41]. Bifurcating solutions of the nonlinear problem were first constructed formally by Malkus and Veronis [57]; the first rigorous proofs of the existence of bifurcating solutions were given by Iudovic [36] and Rabinowitz [69]. Iudovic proved the existence of bifurcating solutions by a topological degree argument. Earlier, however, Velte [99] had proved the existence of branching solutions of the Taylor problem by a topological degree argument.

The infinite plane layer model described here has the advantage that it simplifies the mathematical analysis and gives good quantitative predictions for the onset of convection and of the value of the critical wave number. It has the disadvantage, however, that it allows a high degree of nonuniqueness in the bifurcating solutions. The question then arises as to what mechanisms govern the selection of a particular pattern.

Busse [7], motivated by an earlier work of Malkus, showed that there was a functional associated with the bifurcation problem and argued that this functional would be maximized by the physically correct solutions. Busse's functional is in fact the invariant tensor corresponding to the reduced bifurcation equations, so his method is equivalent to a linearized stability analysis of the bifurcating solutions. Group theoretic ideas are already implicit in Busse's work. In [84], [85] the problem of pattern selection is discussed entirely from a group theoretic point of view.

We begin not with the Boussinesq equations on an infinite plane, but with any general system $G(\lambda, u)$ which is covariant with respect to the group of rigid motions in the plane. For simplicity we consider the case where $u$ is a scalar, though the situation is somewhat more complicated in the case of the Bénard problem. When cellular solutions appear the symmetry is broken from the full group of rigid motions to a crystallographic subgroup. There is so far no mathematical proof that symmetry must be broken in this fashion; 
we simply make the Ansatz that we look for bifurcating doubly periodic solutions. These are characterized by the hypothesis that the solutions be invariant under some subgroup of the full translation group. Let $\Lambda$ be a lattice of vectors in the plane generated as the sum of all integer combinations $\left\{n \omega_{1}+m \omega_{2}\right\}$ for two fixed linearly independent vectors, $\omega_{1}$ and $\omega_{2}$. Let $H(\Lambda)$ denote the subgroup of translations $H(\Lambda)=\left\{T_{\omega}: \omega \in \Lambda\right\}$ where $\left(T_{\omega} u\right)(x)=$ $u(x+\omega)$. We consider the subclass of all functions which are invariant under $H(\Lambda)$. From the translational invariance of $G$, such subclasses are invariant under $G$; so we may restrict ourselves to bifurcation in subspaces of doubly periodic solutions with a given lattice structure. If $\psi$ is $\Lambda$-periodic then $T_{r} \psi$ (where $r$ is a rotation) is $\Lambda$-periodic if and only if $r^{-1}$ leaves $\Lambda$ invariant. We denote by $\mathscr{D}(\Lambda)$ the largest subgroup of $O(2)$ which leaves $\Lambda$ invariant; such a subgroup is called the holohedry. When we restrict ourselves to the bifurcation of $\Lambda$-periodic functions the kernel $\Re$ of $G_{u}\left(\lambda_{c}, 0\right)$ is spanned by the finite set of wave functions

$$
\Re(\Lambda)=\left\{e^{i\langle r \omega, x\rangle}\right\}_{r \in \Phi(\Lambda)}
$$

We denote by $\delta(\Lambda)$ the subgroup of the group of rigid motions which includes all translations plus the elements of $\mathscr{D}(\Lambda) . \mathcal{E}(\Lambda)$ is the symmetry group of the equation $G(\lambda, u)=0$ restricted to $\Lambda$-periodic functions. In the case of the Boussinesq equations the linearized operator $G_{u}$, restricted to the subspace of doubly periodic functions, is a Fredholm operator (Fife [18]).

Let us derive the branching equations for bifurcation of solutions in the hexagonal lattice. The kernel is spanned by the six wave functions

$$
\left\{e^{i\left\langle\omega_{j}, x\right\rangle}, j=1, \ldots, 6\right\}
$$

where the vectors $\omega_{j}$ are as pictured in Figure 9. Denote these wave functions by $\psi_{k}$.

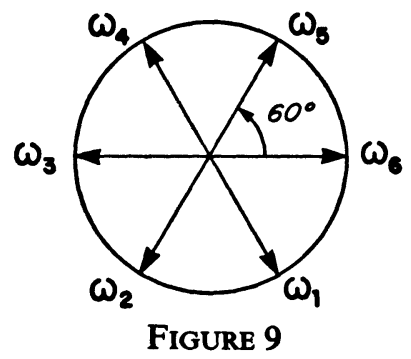

Proposition 15. Let $T_{\sigma}$ be the representation of the group of rigid motions given by $\left(T_{\sigma} u\right)(x)=u\left(\sigma^{-1} \mathbf{x}\right)$. The action $T_{\sigma}$ on the basis functions $\left\{\psi_{1}, \ldots, \psi_{6}\right\}$ is as follows:

(i) If $\sigma=a$ is a translation, $T_{a} \psi_{j}=e^{i\left\langle\omega_{j}, a\right\rangle} \psi_{j}$.

(ii) If $\sigma=r$ is a rotation or reflection in $O(\Lambda)$ then $T_{r}$ acts as a permutation on the $\psi_{j}$. Specifically, let $r(j)$ be the permutation of the vertices $\omega_{j}$ induced by the rotation $r$. Then $T_{r} \psi_{j}=\psi_{r(j)}$.

(iii) $T_{r} \psi_{j}=\bar{\psi}_{j}$ whenever $r \omega_{j}=-\omega_{j}$.

This proposition is easily proved by direct computation. To prove (ii) note that $T_{r} e^{i\langle\omega, x\rangle}=e^{i\langle\omega, r-1 x\rangle}=e^{i\langle r \omega, x\rangle}$ since $r$ is an orthogonal transformation; thus $T_{r}$ moves the wave vector $\omega$ to $r \omega$. 
If instead we write the bifurcation equations in the form

$$
F_{i}\left(\lambda, z_{1}, \ldots, z_{j}\right)=0
$$

then the variables $z_{j}$ should transform as the $\psi_{j}$. Let $\alpha=\left(\begin{array}{lll}1 & 23456\end{array}\right)$ and $\beta=(26)(35)$; these two permutations generate the symmetry group of the hexagon. From $\alpha F=F \alpha$ we have

$$
F_{2}\left(z_{1}, \ldots, z_{6}\right)=F_{1}\left(z_{2}, z_{3}, \ldots, z_{6}, z_{1}\right)
$$

and so forth, so it is only necessary to determine the first component $F_{1}$; the remaining components are obtained by cyclically permuting the variables. From $T_{a} F=F T_{a}$ we get

$$
e^{i\left\langle\omega_{1}, a\right\rangle} F_{1}\left(z_{1}, \ldots, z_{6}\right)=F_{1}\left(e^{i\left\langle\omega_{1}, a\right\rangle} z_{1}, \ldots, e^{i\left\langle\omega_{6}, a\right)} z_{6}\right)
$$

We break $F_{1}$ down into linear, quadratic, cubic terms, etc. If $F_{1}$ contains the linear term $z_{j}$ then $e^{i\left\langle\omega_{1}, a\right\rangle} z_{j}=e^{i\left\langle\omega_{j}, \mathbf{a}\right\rangle} z_{j}$; therefore $F_{1}$ contains no linear terms except $z_{1}$ and $F_{1}=a z_{1}, F_{2}=a z_{2}, \ldots$. Thus the linear term must be a scalar multiple of the identity; and according to Schur's lemma the representation $T_{\sigma}$ restricted to the kernel $\Re(\Lambda)$ is irreducible. The quadratic terms $z_{j} z_{k}$ will be covariant only if

$$
e^{i\left\langle\omega_{1}, \mathbf{a}\right\rangle} z_{j} z_{k}=\left(e^{i\left\langle\omega_{j}, \mathrm{a}\right\rangle} z_{j}\right)\left(e^{i\left\langle\omega_{k}, \mathrm{a}\right\rangle} z_{k}\right)
$$

hence only if $\omega_{1}=\omega_{j}+\omega_{k}$. The only vectors satisfying this condition are $\omega_{j}=\omega_{2}$ and $\omega_{k}=\omega_{6}$, so the only covariant quadratic term is $b z_{2} z_{6}$. Then $F_{2}=b z_{3} z_{1}, F_{3}=b z_{4} z_{2}, \ldots$

In the cubic case we are similarly led to the condition $\omega_{1}=\omega_{j}+\omega_{k}+\omega_{l}$. There are three possible solutions to this equation, leading to the three terms $z_{1}^{2} z_{4}, z_{1} z_{2} z_{5}$, and $z_{1} z_{3} z_{6}$. The symmetry condition $\beta F=F \beta$, however, implies that $F_{1}$ is symmetric in $z_{2}, z_{6}$ and $z_{3}, z_{5}$. Therefore there are really only two covariant cubic terms, namely

$$
c z_{1}^{2} z_{4}+d\left(z_{1} z_{2} z_{5}+z_{1} z_{3} z_{6}\right)
$$

In the other lattices (when the basis vectors $\omega_{1}$ and $\omega_{2}$ make an acute angle other than $\pi / 6$ between each other) there are no quadratic terms but again two cubic terms.

The structure of the bifuration equations can in each case be determined by group-theoretic arguments, and the bifurcation and stability of all possible solutions can be considered for each of the basic lattices. The reduced bifurcation equations (lowest-order terms) can be obtained from the generating functions

$$
\begin{aligned}
\Lambda_{6}: F_{1}\left(\lambda, z_{1}, \ldots, z_{6}\right) & =\lambda z_{1}+a z_{1}^{2} z_{4}+b z_{1}\left(z_{2} z_{5}+z_{3} z_{6}\right), \\
\Lambda_{2}, \Lambda_{4}: F_{1}\left(\lambda, z_{1}, \ldots, z_{6}\right) & =\lambda z_{1}+a z_{1}^{2} z_{3}+b z_{1} z_{2} z_{4} .
\end{aligned}
$$

This is the case when the quadratic term in the $\Lambda_{6}$ case vanishes. $\left(\Lambda_{6}\right.$ denotes the hexagonal, $\Lambda_{4}$ the square, and $\Lambda_{2}$ the rhombic lattices.) If it does not vanish the generating function is

$$
\Lambda_{6}: F_{1}\left(\lambda, z_{1}, \ldots, z_{6}\right)=\lambda z_{1}+c z_{2} z_{6} \text {. }
$$

The other components of the bifurcation equations are obtained by cyclic permutation of the variables $z_{1}, \ldots, z_{6}$. The coefficients $a, b, c$ depend on 
the lattice (in fact on the angle $\theta$ between $\omega_{1}$ and $\omega_{2}$ ) and on the original physical parameters of the problem.

The stability of the bifurcating solutions can be determined in terms of the parameters $a$ and $b$. (By stability here we mean the formal linearized stability of a solution relative to disturbances within the same lattice class. Certainly this is a necessary condition for stability; although a rigorous mathematical treatment of stability on an unbounded domain is so far nonexistent.) In order to obtain any results on pattern selection one must evaluate the dependence of the coefficients $a$ and $b$ on the lattice and on the external parameters of the problem. For the Boussinesq equations there are two physical parameters to the problem, the Rayleigh number and the Prandtl number. For simplicity let us first restrict ourselves to bifurcation of solutions in $\Lambda_{6}$, that is, those which are invariant under translations in the hexagonal lattice. We analyze the linearized stability of the solutions with respect to disturbances in the same invariant subspace, and it can be shown that the pattern selection diagram in Figure 10 holds. Rolls and hexagons bifurcate supercritically provided $a+2 b<0$, but they are not both simultaneously stable; their stabilities depend on the relative sizes of the parameters $a$ and $b$.

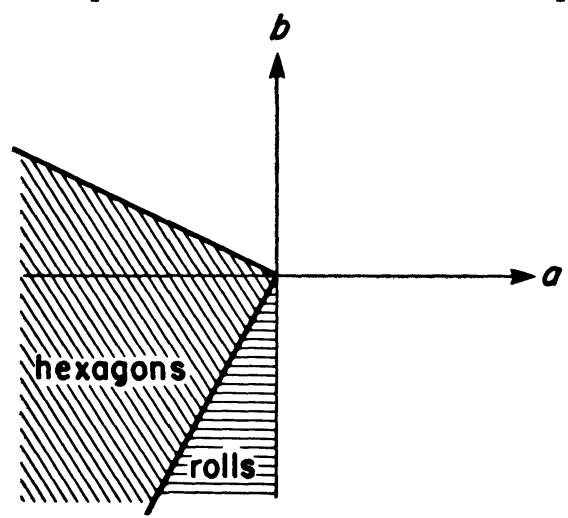

Figure 10

The stability results described above can all be made mathematically rigorous if we restrict ourselves to solutions (and disturbances) in the hexagonal lattice $\Lambda_{6}$. This, of course, does not resolve the issue for the Bénard problem, or other problems where Euclidean invariance is broken, for there is no a priori justification for the restriction to translational invariance under the hexagonal lattice. Nevertheless the analysis demonstrates precisely the concept of selection mechanisms for pattern formation and their relationship to group representation theory. The two parameters $a$ and $b$ in the bifurcation equations signify the existence of two independent covariant mappings of third order for the given representation.

In order to give a complete resolution of the Bénard problem, or more generally the breaking of Euclidean symmetry, one would have to derive selection laws which uniquely select one stable pattern from all possible patterns. This means, in particular, that the stability of patterns in one lattice must be compared to those in another. Partial results of this type can be obtained from purely group-theoretic arguments [85]. 
THEOREM 16. There exists a function $q(\alpha)$ such that

(1) $q(\alpha)=A_{0}+A_{2} \cos 2 \alpha+A_{4} \cos 4 \alpha+\cdots$,

(2) $a=3 q(0), b=6 q(\theta)$

where $\theta$ is the angle between the basic vectors of the lattice and the coefficients $A_{0}, A_{2}, \ldots$ depend only on the basic physical parameters of the problem.

In the case of the Bénard problem, Busse considered a simplified system of equations obtained by letting the Prandtl number go to infinity in the Boussinesq equations. As a consequence, for the simplified system, one can show that $q(\alpha)=A+B \cos 2 \alpha$, and then the pattern selection diagram in Figure 11 is valid.

If the quadratic term does not vanish in the hexagonal case (which happens if the material properties such as viscosity, specific heat, or thermal diffusivity

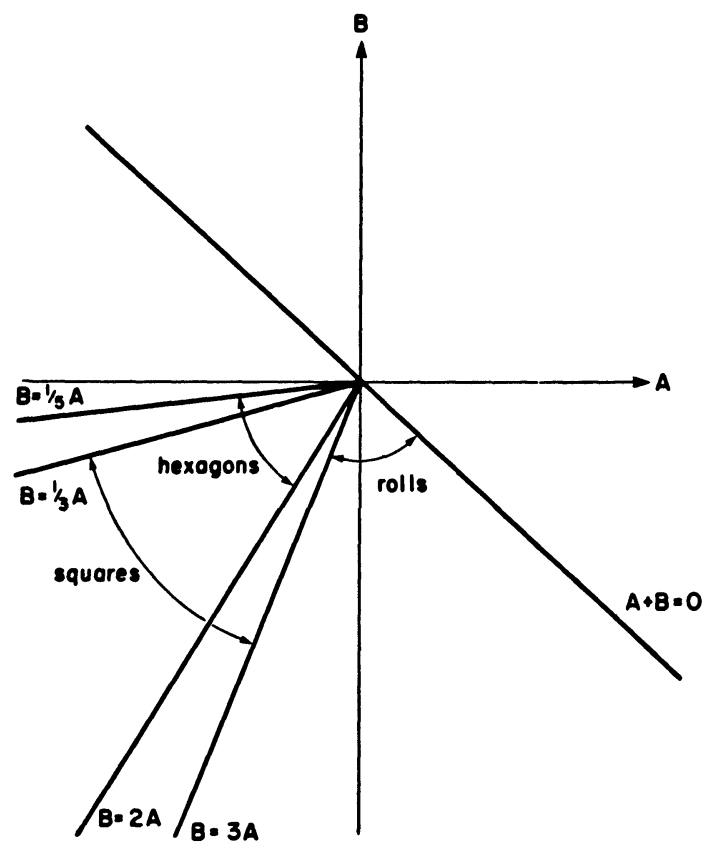

FIGURE 11.

In the sector between the lines $B=3 A$ and $A+B=0$, rolls are the only possible doubly period solutions which can be stable. That is, if for a given set of external physical conditions the pair $(A, B)$ lies in this sector, then rolls are the only solutions which can be stable when tested against all disturbances in the three lattice classes. In the sector $2 A<B$ $<\frac{1}{3} A<0$ hexagons may be stable while rolls are unstable. Squares, however, are also stable in this region, as are rectangles in the rectangular lattices with angle $\theta>\pi / 3$. In general, $\theta$-rectangles may be stable in the sector

$$
A(1-2 \cos 2 \theta)<B<-\left(\frac{1+2 \cos 2 \theta}{3}\right) A .
$$


vary, when the density-temperature relation is nonlinear, or when surface tension drives the convection) then one obtains the branching of subcritical solutions in the hexagonal lattice. These solutions are invariant under the entire symmetry group of the hexagon and are unstable on both sides of criticality. The eigenvalues of the linearized equations about the bifurcating solution are, up to a scalar multiple at lowest order, $-1,3,2,2,0,0$. The two zero eigenvalues arise because of the presence of the two-parameter translation group. The fact that one eigenvalue is negative means there is one unstable mode on the subcritical branch. These results can be obtained entirely by group theoretic arguments. Since the subcritical branch has one unstable mode it can "bend back" and regain stability, as sketched in Figure 4. There is, of course, no general proof that this branch must bend back, as in the diagram.

It may be useful to compare the foregoing analysis with the earlier work on bifurcating solutions of the Bénard problem by Iudovic [36] and Rabinowitz [69]. These authors construct doubly-periodic solutions by symmetrizing within a given, fixed lattice class but without regard to the stability of the solutions. Within a given lattice they considered the subspace of functions which are completely invariant under the symmetry group of the lattice, on which the branch point is simple. But at a simple eigenvalue, as we have seen, supercritical solutions are stable and subcritical solutions are unstable, so when the analysis is carried out in this fashion one is led to the erroneous conclusion that any solution which bifurcates supercritically is stable. Altogether different conclusions are obtained, and a mechanism for pattern selection is possible, if the symmetrization argument is avoided.

Busse, in his work, does not a priori make the symmetrization argument, but, due to other assumptions, he is led to the conclusion that only rolls can be stable. I believe that a valid theoretical explanation of the mechanisms for pattern selection does not yet exist.

I want to emphasize that I do not claim the Bénard problem to be resolved by the above considerations, though I do think the analysis sheds some light on the complexity of the problem. In any event, the group-theoretic approach to a bifurcation problem is very nicely illustrated by the Bénard problem.

5. Bifurcation in the presence of the rotation group. Now let us consider the derivation of the bifurcation equations when the symmetry group in question is $O(3)$, the group of rotations of the sphere in $\mathbf{R}^{3}$. Busse [8] was the first person to consider a general bifurcation problem in the presence of $O(3)$. Such bifurcation problems arise, for example, in the onset of convection in a spherical mass or the buckling of a perfectly uniform spherical shell. In these cases, complete rotational symmetry is broken by the bifurcating solutions. The irreducible representations of $S O(3)$ are denoted by $D^{l}$ for $l=$ $0,1,2, \ldots$ and are of dimension $2 l+1$. They arise when one considers the transformation properties of the spherical harmonics $Y_{m}^{l}(\theta, l)=$ $P_{l, m}(\cos \theta) e^{i m l},-l<m<l$. Let the infinitesimal generators of $S O(3)$ be $L_{1}, L_{2}$, and $L_{3}$; these satisfy the commutation relations

$$
\left[L_{i}, L_{j}\right]=\varepsilon_{i j k} L_{k}
$$

where $\varepsilon_{i j k}$ is the completely antisymmetric tensor. Putting $J_{ \pm}= \pm L_{2}+i L_{1}$, 
$J_{3}=-i L_{3}$ we obtain for $J_{1}, J_{2}$, and $J_{3}$ the commutation relations

$$
\left[J_{+}, J_{-}\right]=2 J_{3}, \quad\left[J_{3}, J_{ \pm}\right]= \pm J_{ \pm} .
$$

LEMMA 17. Let $V$ be a real vector space which transforms irreducibly under the rotation group according to the representation $D^{l}$. Then there exists a basis $\left\{f_{m}\right\}$ for the complexification of $V$ such that

$$
\begin{gathered}
J_{3} f_{m}=m f_{m}, \\
J_{ \pm} f_{m}=\beta_{ \pm m} f_{m \pm 1},
\end{gathered}
$$

$-l \leqslant m \leqslant l$ and $\beta_{m}=\sqrt{(l-m)(l+m+1)}$. In addition, the $f_{m}$ can be normalized so that

$$
\bar{f}_{m}=(-1)^{m} f_{-m} \text {. }
$$

The relations (7a), (7b) can be derived entirely from the commutation relations (6) by standard methods [64]. We shall work with representations of $O(3)$ such that $D^{\prime}(R)=-D^{l}(R)$ for a reflection of $R$ (see [64]).

Let $\Re$ be the kernel of the linearized operator $L_{0}=G_{u}\left(\lambda_{c}, 0\right)$. Identify $\Re$ with linear polynomials in the variables $z_{-l}, \ldots, z_{l}$ which transform under the Lie algebra according to (7). The algebra $K\left[z_{-l}, \ldots, z_{l}\right]$ of polynomials in the variables $z_{-l}, \ldots, z_{l}$ is isomorphic to the algebra of symmetric tensors over $\Re$. Extend the operators $J_{3}, J_{ \pm}$to be derivations on the algebra $K$ :

$$
J(\alpha f+\beta y)=\alpha J f+\beta J g, \quad J(f g)=(J f) g+f(J g)
$$

where $\alpha, \beta$ are scalars and $f, g$ are polynomials in $K$. Let the bifurcation equations be

$$
F_{m}\left(\lambda, z_{-l}, \ldots, z_{l}\right)=0 .
$$

These will be covariant with respect to $D^{\prime}$ provided the $F_{m}$ transform as the $z_{m}$; that is,

$$
J_{3} F_{m}=m F_{m}, \quad J_{ \pm} F_{m}=\beta_{ \pm m} F_{m \pm 1}
$$

where $J_{3}, J_{+}$, and $J_{-}$act as derivations on $F_{m}$. For example, the quadratic polynomials $F_{m}$ are obtained as follows. The action of $J_{3}$ on $z_{j} z_{k}$ is

$$
J_{3}\left(z_{j} z_{k}\right)=\left(J_{3} z_{j}\right) z_{k}+z_{j}\left(J_{3} z_{k}\right)=(j+k) z_{j} z_{k}
$$

so $J_{3} z_{j} z_{k}=m z_{j} z_{k}$ if and only if $j+k=m$. Therefore

$$
F_{m}=\sum_{m_{1}+m_{2}=m} a_{m_{1} m_{2} m} z_{m_{1}} z_{m_{2}}
$$

In particular, when $l$ is even,

$$
F_{l}=a_{0} z_{l} z_{0}+a_{1} z_{l-1} z_{1}+\cdots+a_{l / 2}\left(z_{l / 2}\right)^{2} .
$$

Furthermore, $J_{+} F_{l}=\beta_{l} F_{l}=0$ and this condition gives us a set of linear equations for the coefficients $a_{0}, \ldots, a_{l / 2}$. In the case $l=2$,

$$
\begin{aligned}
F_{2} & =a z_{2} z_{0}+b z_{1}^{2}, \\
J_{+} F_{2} & =a \beta_{0} z_{2} z_{1}+2 b \beta_{1} z_{1} z_{2}=\left(a \beta_{0}+2 b \beta_{1}\right) z_{1} z_{2}=0,
\end{aligned}
$$


so $a \beta_{0}+2 b \beta_{1}=0$. The last equation determines the coefficients $a$ and $b$, hence $F_{2}$, up to a scalar multiple. Once $F_{l}$ is known we get $F_{l-1}$ from

$$
J_{-} F_{l}=\beta_{-l} F_{l-1}
$$

and so forth. In this way we construct all the $F_{m}$ 's.

This procedure extends immediately to higher-order terms. For example, to get third-order terms we write

$$
F_{l}=\sum_{i+j+k=l} a_{i j k} z_{i} z_{j} z_{k}
$$

and apply $J_{+} F_{l}=0$ to get a linear system of equations for the $a_{i j k}$. For $l=1$ there is only one solution but for $l=3$ there are two independent solutions [86].

For even $l$ the quadratic terms of the covariant mapping are given by

$$
F_{m}=\sum_{m_{1}+m_{2}=m}(-1)^{m}\left(\begin{array}{ccc}
l & l & l \\
m_{1} & m_{2} & -m
\end{array}\right) z_{m_{1}} z_{m_{2}}
$$

where

$$
\left(\begin{array}{lll}
j_{1} & j_{2} & j_{3} \\
m_{1} & m_{2} & m_{3}
\end{array}\right)
$$

are the Wigner $3 j$ symbols for the rotation group. For odd $l$ the covariant quadratic mappings are antisymmetric, and one must go to third-order terms in the bifurcation equations. For even $l$ the quadratic terms possess a gradient structure, as follows. Consider the homogeneous polynomial of degree 3 ,

$$
p\left(z_{-l}, \ldots, z_{l}\right)=\frac{1}{3} \sum_{-l}^{l} F_{m} \bar{z}_{m},
$$

restricted to the real subspace of $\Re$ for which $\bar{z}_{m}=(-1)^{m} z_{-m}$. There we have

$$
\begin{aligned}
p\left(z_{-l}, \ldots, z_{l}\right) & =\frac{1}{3} \sum_{-l}^{l}(-1)^{m} F_{m} z_{-m} \\
& =\frac{1}{3} \sum_{m_{1}, m_{2}, m_{3}=-l}\left(\begin{array}{ccc}
l & l & l \\
m_{1} & m_{2} & m_{3}
\end{array}\right) z_{m_{1}} z_{m_{2}} z_{m_{3}} .
\end{aligned}
$$

For even $l$ the $3_{j}$ symbols are completely symmetric in $m_{1}, m_{2}, m_{3}$ and therefore

$$
\frac{\partial p}{\partial z_{m}}=F_{m}\left(z_{-l}, \ldots, z_{l}\right)
$$

Therefore the reduced bifurcation equations (linear plus quadratic terms) take the form

$$
\sigma z_{m}+\frac{\partial p}{\partial z_{m}}=0
$$

which are the Euler-Lagrange equations for the variational problem $\operatorname{Max}_{|z|=1} p$ where $|z|^{2}=\Sigma_{-l}^{l}(-1)^{m} z_{m} z_{-m}$. The function $p$ is the third-order invariant for $D^{l}$; that is, $p\left(D^{\prime} z\right)=p(z)$ and the Euclidean norm $|z|^{2}$ is the second-order invariant. 
Leon Green and I have succeeded in casting this variational problem in a slightly different way. For even $l$ we have the Clebsch-Gordon series

$$
D^{l / 2} \otimes D^{l / 2}=D^{l} \oplus D^{l-1} \oplus \cdots \oplus D^{0}
$$

and the associated representation

$$
U_{g} A=D^{1 / 2}(g) A D^{1 / 2}\left(g^{-1}\right)
$$

on $(l+1) \times(l+1)$ matrices $A$. This representation is unitary relative to the inner product

$$
\langle A, B\rangle=\frac{1}{2} \operatorname{tr} A B^{*}
$$

where $B^{*}$ is the Hermitian conjugate of $B$. The third-order invariant (there is only one since $D^{l} \otimes D^{l} \otimes D^{l}$ contains $D^{0}$ only once) is

$$
p(A)=\frac{1}{3} \operatorname{tr} A^{2} A^{*} \text {. }
$$

The highest weight space, the one that transforms like $D^{l}$ in (8) consists of Hermitian symmetric matrices, so we may rephrase our variational problem as $\operatorname{Min} \frac{1}{3}$ tr $\boldsymbol{A}^{3}$ subject to

$$
\frac{1}{2} \operatorname{tr} A^{2}=1 \text { and } \operatorname{tr} A B_{j}=0
$$

where the $B_{j}$ are symmetric matrices which lie in the lower weight invariant subspaces. In particular, $\operatorname{tr} A I=\operatorname{tr} A=0$. For $l=2(9)$ reads $D^{1} \otimes D^{1}=$ $D^{2} \oplus D^{1} \oplus D^{0}$ but the matrices transforming as $D^{1}$ are antisymmetric; so we have only the constraints $\operatorname{tr} A=0, \operatorname{tr} A^{2}=2$, and the Euler-Lagrange equations are $A^{2}=\lambda A+\gamma I$ where $A$ and $I$ are $3 \times 3$ matrices. (The gradient of the functional $\frac{1}{3} \operatorname{tr} A^{3}$ is the mapping $A \rightarrow A^{2}$.) These equations can be completely solved as follows.

Taking the trace we get $\gamma=\frac{2}{3}$. When $l=2, A$ is a $3 \times 3$ symmetric matrix, and we can choose a rotation $g$ such that $D^{1}(g) A D^{1}\left(g^{-1}\right)$ is diagonal, since $D^{1}(g)$ ranges over all orthogonal matrices as $g$ ranges over $O(3)$. So, assuming $A$ is diagonal, we arrive at the equations

$$
\mu_{i}^{2}=\lambda \mu_{i}+\frac{2}{3}, \quad i=1,2,3
$$

where $\mu_{i}$ are the eigenvalues of $A$. The constraints are

$$
\mu_{1}^{2}+\mu_{2}^{2}+\mu_{3}^{3}=2, \quad \mu_{1}+\mu_{2}+\mu_{3}=0 .
$$

There are two sets of solutions to these equations, namely,

$$
\lambda=-\frac{1}{\sqrt{3}}, \quad A=\left(\begin{array}{ccc}
\frac{1}{\sqrt{3}} & 0 & 0 \\
0 & \frac{1}{\sqrt{3}} & 0 \\
0 & 0 & -\frac{2}{\sqrt{3}}
\end{array}\right)
$$


and

$$
\lambda=\frac{1}{\sqrt{3}}, \quad A=\left[\begin{array}{ccc}
\frac{1}{\sqrt{3}} & 0 & 0 \\
0 & -\frac{1}{\sqrt{3}} & 0 \\
0 & 0 & \frac{2}{\sqrt{3}}
\end{array}\right] .
$$

The order of the eigenvalues of the diagonal of $A$ is immaterial, for any permutation of the diagonal entries of $A$ produces a point on the same orbit. Indeed, any such permutation is accomplished by the operation $P A P^{-1}$, where $P$ is a permutation matrix, and such a $P$ is an element for $O(3)$. One of the orbits above gives the maximum of the functional $\frac{1}{3} \operatorname{tr} A^{3}$ on the sphere $\frac{1}{2} \operatorname{tr} A^{2}=1$; the other orbit gives the minimum. The isotropy subgroup in each case is $O(2)$ (rotations which leave

$$
\left(\begin{array}{l}
0 \\
0 \\
1
\end{array}\right)
$$

invariant), so each extremal is axisymmetric. The case $l=2$ was first treated by Busse by another method. The above method, while quite straightforward in the case $l=2$, becomes extremely complicated already in the case $l=4$ and so does not seem to be a practical approach to the resolution of the bifurcation equations in the general case.

Busse has found a series of special solutions of the reduced bifurcation equations for all even $l$. These solutions belong to one of two special classes:

$$
\begin{array}{llll}
z_{0} & \neq 0, & z_{n}, z_{2 n} \neq 0, \quad \frac{1}{3} l<n<\frac{1}{2} l, & z_{m}=0 \text { otherwise; } \\
z_{0} \neq 0, & z_{n} \neq 0 \text { for a single } n>l / 2, & z_{m}=0 \text { otherwise. }
\end{array}
$$

The axisymmetric solutions never give a minimum to the variational problem except in the case $l=2$.

The stability analysis of the bifurcating solutions yields the following result [77], [86], [87].

THEOREM 18. Suppose the reduced bifurcation equations have a gradient structure and that a solution $v_{0}$ is obtained as a maximum of the corresponding variational problem. Then one eigenvalue of the Jacobian $Q_{\xi}\left(\sigma_{0}, v_{0}\right)$ is negative and the rest are nonnegative.

Accordingly, from Corollary 5, it follows that the corresponding bifurcating solution has one subcritical unstable mode (the branch is transcritical, that is, it appears as in Figure 4). Thus, for $l$ even the bifurcating solutions are unstable on both sides of the branch point. The solution branch with one unstable mode, however, may "bend back and regain stability", as indicated in Figure 4. There is no general theorem to this effect, but it is a common feature of nonlinear problems. In such a situation there exists the possibility of a discontinuous jump from the symmetric (rotationally-invariant) state to the nontrivial branch possessing a smaller symmetry group. Such a situation 
in an elastic structure would take the form of a sharp buckling below the critical parameter value (at subcritical loads).

6. Bifurcation of waves. We have seen how group-theoretic methods can be applied to analyze the bifurcation of stationary solutions at a multiple eigenvalue when the multiplicity is due to symmetry. The same methods are applicable when eigenvalues cross the imaginary axis but are multiple due to spatial symmetries in the problem. In this case we should expect to see symmetry breaking in space-time, which would manifest itself as the onset of wave-like phenomena. The relevant invariance group would be the group $G \times \mathbf{R}^{+}$where $G$ is the group of spatial symmetries and $\mathbf{R}^{+}$is the group of time translations $t \rightarrow t+\delta$ (but not $t \rightarrow-t$ ). This is the natural generalization of the Hopf bifurcation theorem to group-invariant problems.

The analysis of bifurcating waves at the onset of instability has at this point not yet been fully developed, although there has been some initial work on the subject. Turing [96] discusses the possibility of traveling wave solutions to a particular system of reaction and diffusion equations on a circle. Such solutions have been obtained in a model chemical reaction analyzed by Auchmuty and Nicholis [3]. Both rotating and standing wave solutions have been obtained by Erneux and Herschkowitz-Kaufman [27].

J. T. Stuart formally obtained traveling wave solutions (Tollmien-Schlicting waves) in his analysis of the onset of instability in plane Poiseuille flow [90]. Auchmuty [1] has obtained rigorous results on the bifurcation of waves in circular geometries.

To illustrate the use of group-theoretic methods in the analysis of bifurcating waves let me take the simple problem treated by the aforementioned authors and derive the structure of the bifurcation equations. Consider a bifurcation problem which is invariant under space and time translations and spatial reflections. The group operations are $T_{\gamma} x=x+\gamma, R x=-x$, and $S_{\delta} t=t+\delta$. If the null functions have wave number $k$ and frequency $\omega$ then the null space for the linear operator is four dimensional and is spanned by $\psi_{1}=e^{i(k x+\omega t)}, \psi_{2}=e^{-i(k x+\omega t)}, \psi_{3}=e^{+i(k x-\omega t)}, \psi_{4}=e^{-i(k x-\omega t)}$. Relative to $\psi_{1}, \ldots, \psi_{4}$ the group operations have the matrix representations

$$
\begin{aligned}
& T_{\gamma}=\left[\begin{array}{cccc}
e^{i k \gamma} & & & 0 \\
& e^{-i k \gamma} & & \\
& 0 & e^{i k \gamma} & \\
& 0 & e^{-i k \gamma}
\end{array}\right], \quad S_{\delta}=\left[\begin{array}{cccc}
e^{i \omega \delta} & & & \\
& e^{-i \omega \delta} & & \\
& & e^{-i \omega \delta} & \\
& & & e^{i \omega \delta}
\end{array}\right] \\
& R=\left[\begin{array}{lll}
0 & 1 \\
1 & 1 & 0
\end{array}\right]
\end{aligned}
$$

The problem can be reduced by the Lyapounov-Schmidt method to a system of bifurcation equations, and these must take the form

$$
F_{i}\left(\lambda, \omega, z_{1}, \ldots, z_{4}\right)=0, \quad i=1,2,3,4 .
$$

Since we are looking for real solutions we want $z_{2}=\bar{z}_{1}, z_{4}=\bar{z}_{3}$ and $F_{2}=\bar{F}_{1}$, 
$F_{4}=\bar{F}_{3}$. The symmetry $R F=F R$ leads to

$$
\begin{aligned}
& F_{4}\left(\lambda, \omega, z_{1}, \ldots, z_{4}\right)=F_{1}\left(\lambda, \omega, z_{4}, z_{3}, z_{2}, z_{1}\right), \\
& F_{3}\left(\lambda, \omega, z_{1}, \ldots, z_{4}\right)=F_{2}\left(\lambda, \omega, z_{4}, z_{3}, z_{2}, z_{1}\right) .
\end{aligned}
$$

Therefore it is enough to find $F_{1}$, for the other components can be found by applying $R$ or taking complex conjugates.

Suppose $F_{1}$ contains a term $z_{1}^{z} z_{2}^{b} z_{3}^{c} z_{4}^{d}$. The symmetry $T_{\gamma} F=F T_{\gamma}$ implies

$$
e^{i k \gamma}\left(a_{1}^{a} z_{2}^{b} z_{3}^{c} z_{4}^{d}\right)=\left(e^{i k \gamma} z_{1}\right)^{a} \cdots=e^{i k \gamma(a-b+c-d)}\left(z_{1}^{a} z_{2}^{b} z_{3}^{c} z_{4}^{d}\right)
$$

hence $a-b+c-d=1$. Similarly the symmetry $S_{\delta} F=F S_{\delta}$ leads to $a-b$ $-c+d=1$. Combining these two equations we get $a=b+1, c=d$ so $F_{1}$ has the general term $z_{1}\left(z_{1} z_{2}\right)^{b}\left(z_{3} z_{4}\right)^{d}$, or $z|z|^{2 b}|w|^{2 d}$ where $z=z_{1}$ and $w=z_{3}$. The most general $F_{1}$ is therefore

$$
F_{1}(\lambda, \omega, z, \bar{z}, \omega, \bar{\omega})=z g\left(\lambda, \omega,|z|^{2},|\omega|^{2}\right) .
$$

Then $F_{4}$ must be of the form

$$
F_{4}(\lambda, \omega, z, \bar{z}, \omega, \bar{\omega})=\omega g\left(\lambda, \omega,|\omega|^{2},|z|^{2}\right) .
$$

The other two equations are complex conjugates of these so nothing new is obtained, and the bifurcation equations are

$$
z g\left(\lambda, \omega,|z|^{2},|\omega|^{2}\right)=0, \quad w g\left(\lambda, \omega,|w|^{2},|z|^{2}\right)=0
$$

and the elements of the null space are of the form $z \psi_{1}+\bar{z} \psi_{2}+w \psi_{3}+\bar{w} \psi_{4}=$ $2 \operatorname{Re}\left[z \psi_{1}+w \psi_{3}\right]$. There are two basic solution types:

(i) $|z|=|w|$ and $g\left(\lambda, \omega,|z|^{2},|z|^{2}\right)=0$;

(ii) $w=0$ and $g\left(\lambda, \omega,|z|^{2}, 0\right)=0$.

The case $z=0$ is equivalent to $w=0$ under the reflection $R$.

If $|z|=|w|$ we take $z>0$ by a suitable translation in $x$ and then $w=z e^{i \gamma}$. Then the solution to first order is

$$
z\left(\psi_{1}+\psi_{2}+e^{i \gamma} \psi_{3}+e^{-i \gamma} \psi_{4}\right)=2 z(\cos (k k+\omega t)+\cos (k x-\omega t+\gamma)) .
$$

By suitable translations in $x$ and $t$ this is equivalent to $4 z \cos k x \cos \omega t$; these waves appear as standing waves. In case (ii) with $w=0$ we get $\psi_{1}+\psi_{2}=$ $2 \cos (k x+\omega t)$, which are traveling waves. The possibility of two distinct modes of bifurcating waves was observed by Erneus and Herschkowitz-Kaufman and has also been discussed by Cowan and Ermentrout [12].

A stability theory has not yet been worked out for such bifurcating waves, but a stability analysis may produce a mechanism which will select one or the other mode of propagation, depending on the sizes of other parameters in the problem.

Symmetry breaking in natural phenomena. In this section I wish to discuss a variety of scientific disciplines in which spontaneous symmetry-breaking manifests itself as a primary feature of the problem.

1. Morphogenesis. A striking example of symmetry breaking in a biological system is the breakdown of rotational symmetry in the Fucus seaweed egg [53]. At a critical stage in the development of the egg a transition is made from a spherically symmetric membrane potential distribution to a polarized state with an axial symmetry, and a net transcellular current leaving one pole 


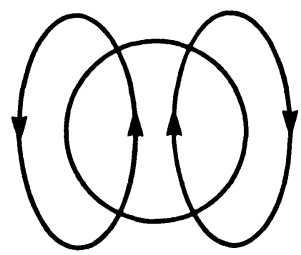

Figure 12

\section{Self-electrophoresis currents in the Fucus egg}

and entering the opposite. This phenomenon is termed "self-electrophoresis". According to Larter and Ortoleva [53], who are conducting a theoretical investigation of this effect, "The net transcellular potential gradient is believed to be essential in the development of the asymmetry which leads to dramatically different rhizoid and thallus cells after the first division of the egg". Larter and Ortoleva base their theory on models which allow for the full electrochemical nature of cellular phenomena. For slow processes these equations reduce to Poisson's equation and a system of rate-reaction equations:

$$
\begin{aligned}
\Delta V & =\frac{-4 \pi \mathcal{F}}{\varepsilon} \sum_{j=1}^{n} z_{j} C_{j}, \\
\frac{\partial C}{\partial t} i & =\operatorname{div}\left\{\sum_{j=1}^{n} \mathscr{D}_{i j} \operatorname{grad} C_{j}+M_{i j} C_{j} \operatorname{grad} V\right\}+G_{i}(C)
\end{aligned}
$$

where $\mathcal{F}$ is Faraday's constant, $\varepsilon$ the dielectric constant, $C_{i}$ are species concentrations, $z_{i}$ are valences, $\mathscr{D}_{i j}$ is a matrix of diffusion coefficients, $M_{i j}$ a matrix of mobilities, and $G_{i}(C)$ are chemical reaction rate terms.

The symmetry breakdown in the Fucus egg is of the form rotational invariance to axial invariance. That is, prior to self-electrophoresis the solutions are invariant under the entire rotation group $O(3)$, while the bifurcating solutions are invariant only under a subgroup of rotations about a fixed axis. The solutions thus appear in two-dimensional orbits with one-dimensional isotropy subgroup. This, however, is by no means the only symmetry breakdown which can occur in rotationally-invariant systems; and more complicated symmetry breakdowns are relevant in geophysical problems.

2. Dynamo problem. One problem in which nonaxisymmetric solutions are of interest is the Dynamo problem: By what mechanism is the earth's magnetic field sustained? The current consensus among geophysicists is that the magnetic field is sustained by electric currents flowing in the electrically conducting earth's core which interact with the convective fluid motions of that core. It is known (Cowlings theorem) that purely axisymmetric magnetic fields cannot satisfy the dynamo equation. The dynamo process in generators depends on a multiply-connected distribution of electrical conductivity, which permits a simple rotational motion. The uniform conductivity of a homogeneous, spherical dynamo may require a more complex velocity field for dynamo action. Thus the question of nonaxisymmetric flow patterns appearing at the onset of convection becomes extremely interesting for the Dynamo problem. The equations of dynamo theory are the convection 
equations plus an equation governing the magnetic field:

$$
\begin{aligned}
\frac{\partial \vec{u}}{\partial t}+(\vec{u} \cdot \nabla) \vec{u}+2 \vec{\Omega} \times \vec{u} & =-\nabla p-\beta \vec{g} \theta+\nu \Delta \vec{u}+\frac{1}{\mu}(\nabla \times \vec{B}) \times \vec{B},(12 \mathrm{a}) \\
\operatorname{div} \vec{u} & =0 \\
\frac{\partial \theta}{\partial t}+(\vec{u} \cdot \nabla) \theta & =-u \cdot \nabla T_{0}+\kappa \Delta \theta \\
\frac{\partial \vec{B}}{\partial t} & =\operatorname{curl}(\vec{u} \times \vec{B})+\eta \Delta \vec{B}
\end{aligned}
$$

where $T_{0}$ is the base temperature profile in the absence of convection and $2 \vec{\Omega} \times \vec{u}$ is the Coriolis term.

When no magnetic field is present the quadratic term $(\nabla \times \vec{B}) \times \vec{B}$ in the first equation vanishes, and we have a pure convection problem. When the convective velocity $\vec{u}$ reaches a magnitude and configuration which can sustain a growing magnetic field in (12d) then a bifurcation may take place and a nontrivial magnetic field may be sustained. Equation (12d) is linear so for a given $\vec{u}$ one should expect an exponentially growing magnetic field $\vec{B}$; but the nonlinear coupling between $\vec{B}$ and $\vec{u}$ via (12a) may be expected to prevent unlimited growth, so that a stable equilibrium is attained. Since (12d) is homogeneous in $\vec{B}$ some external magnetic field (for example, the Sun's) is required to "seed" the dynamo process. For further discussion and a survey of the literature, see the articles by Busse [9] and Roberts [72].

3. Wave propagation in neural networks. Bifurcation phenomena in simple mathematical models of excitatory inhibitory neuro-networks have been discussed recently by Cowan and Ermentrout [12]. Neural networks are aggregates of nerve cells which interact with other neurons in the network in either an excitatory or inhibitory way, and so it is plausible to expect these networks to exhibit such nonlinear collective phenomena as bifurcation, threshold effects, and hysteresis. Cowan and Ermentrout model these networks by a system of equations

$$
\mu Y=-Y+S(K Y+P)
$$

where $Y$ is a two-component vector, $S$ is a nonlinear vector-valued function, $K$ is a linear convolution operator, and $P$ is the external stimulus. Equation (13) may be studied in one, two, or three dimensions.

Cowan and Ermentrout seek to model the patterns of activity of the central nervous system by showing how organized space-time neuronal activity patterns can arise through the mechanisms of bifurcation from an initially uniform resting state. They investigate the structure of the bifurcation point when two pairs of complex conjugate eigenvalues cross the imaginary axis simultaneously. In that case one gets secondary bifurcation as some of the parameters in the problem are varied.

Cowan and Ermentrout have also treated hallucinatory phenomena from the standpoint of symmetry breaking bifurcations [13]. Recent experiments on mescaline induced hallucinations have led to the conclusion that most simple hallucinations could be classified into one of four categories: "(a) grating, lattice, honeycomb or chessboard; (b) cobweb; (c) funnel, tunnel, cone or 
vessel; (d) spiral". Cowan and Ermentrout base their analysis on the contention that "simple formed hallucinations arise from an instability of the resting state leading to concomitant spatial patterns of activity in the cortex. This instability arises from a combination of enhanced excitatory modulation and decreased inhibition. [We] demonstrate that such spatial patterns are a property of neural nets with strong lateral interactions acting to provide a dominant negative feedback. [We] formalize these postulates into a simple mathematical model and then use bifurcation theory to demonstrate the existence of the relevant spatial patterns".

The relevant spatial patterns are none other than those crystallographic patterns which have already made their appearance in the Benard problem, with one additional factor. Experimental observations have established that in primates there is a conformal transformation from the retinal field, which is circular, to the cortical field, which has Cartesian (rectangular) symmetry. This implies that the transformation from retinal polar coordinates to cortical rectangular coordinates must be essentially logarithmic in nature. Such a logarithmic transformation would take a tunnel pattern consisting of concentric circles of activity to a pattern of rolls parallel to the $y$ axis. Similarly, spirals are transforms of rolls with some other direction. Thus the patterns observed in hallucinatory phenomena are images under the log transformations of the cellular patterns familiar in the analysis of the Bénard problem: hexagons, squares, rectangles, and rolls. Cowan and Ermentrout then assume that, as some parameter $\lambda$ increases, the strength of the excitation increases until, beyond some critical value $\lambda_{0}$, the rest state becomes unstable and gives way to the stationary patterns of spatial activity. Thus, according to their theory, the drug-induced hallucinatory patterns are precisely those which one would see when Euclidean invariance is broken.

4. Phase transitions in statistical mechanics. The notion of symmetry breaking is fundamental to phase transitions, yet much harder to treat mathematically. Until the renormalization theories developed in recent years, the primary approach to phase transitions was, in one way or another, a meanfield approximation coupled with a bifurcation analysis of the mean-field equations. The simplest mean-field theories for critical phenomena were the scalar equations of state, such as the Van der Waals equation for a gas of the Curie-Weiss model for a ferromagnet. In more elaborate theories the state of the ensemble is described, for example, by a single particle density function, and an integral equation is derived for this function by some kind of closure hypothesis for the hierarchy of higher-order (multiple particle) correlation functions. This line of analysis was first pursued by Kirkwood and Monroe, [48] and more recently by Raveche and Stuart [70], and Rice and his coworkers. (See [50], [101].) Nevertheless, these approximations are still mean-field theories, and depend, for their validity, on the assumption that fluctuations are negligible; the major difficulty is that in many cases, large fluctuations become important precisely at the critical point. In fact, at a critical point the fluctuations very often diverge to infinity, making the mean-field approximation invalid, and it is this fact which accounts for the deviation of the critical exponents from the "classical exponents" predicted by bifurcation (mean-field) models. All this notwithstanding, the bifurcation 
models do have some areas of validity, and are generally successful in predicting the symmetry changes actually observed. Landau's [6], [56] theory of second-order phase transitions is a phenominological description of phase transitions which is essentially a theory of "symmetry-breaking bifurcations".

This point of view has been expressed very concisely by Tareva and Trapezina [92], [93]: “The generalized mean-field approximation usually brings us to the formulation of the broken-symmetry problem in terms of the bifurcation on a nonlinear integral equation solution for the Bogolyubov quasi-average. Particularly in a number of papers the liquid-solid phase transition is considered as a bifurcation of the solution of the equation of Hammerstein type

$$
\Phi\left(r_{1}\right)-\mu \int K\left(r_{1}, r_{2}\right) f\left(\Phi\left(r_{2}\right), r_{2}\right) d r_{2}=0 \%
$$

The phase transitions of the ensemble are described in terms of bifurcations of this integral equation. See also the review article by Kozak [50].

In the area of nonequilibrium thermodynamics the operation of the laser can be described by a mean-field theory which is amenable to a bifurcation analysis. In the Dicke-Haken-Lax model of the laser it is possible to describe the many body photon field by a mean-field theory as $N$ (the number of degrees of freedom) tends to infinity. Thus it is possible in this case to solve a nonlinear quantum mechanical model, far from equilibrium, by reducing the problem to a system of ordinary differential equations for the expectation values of the extensive variables. (See Haken [23], and Hepp and Lieb [26].) The onset of laser action in these theories is then described by the bifurcation of time-periodic solutions from the equilibrium solution, that is, so-called Hopf bifurcation.

5. Symmetry breaking in elementary particle physics. The notion of "spontaneous symmetry breaking" plays a fundamental role in quantum field theory. It is not possible for me to discuss this in depth here, but let me briefly describe the work of Michel and Radicati [61], [62], [63] in symmetry breaking in elementary particle theory. They study the adjoint action of $S U(3)$ on the vector space $Q$ of traceless Hermitian matrices $A$. The inner production on $Q$ is $(A, B)=\frac{1}{2} \operatorname{tr} A B^{*}$, and the group action is $A \rightarrow U A U^{*}$ where $U \in S U(3)$. This action leaves the inner product invariant. There are two third-order invariants of this action, namely

$$
\{A, B, C\}=\frac{1}{2} \sqrt{3} \operatorname{tr}(A B+B A) C, \quad\{A, B, C\}=\frac{-i}{2} \operatorname{tr}[A, B] C
$$

where $\{,$,$\} is completely symmetric and [,$,$] is antisymmetric. These two$ third-order invariants induce two algebras on $Q$ via the following device. The form $C \rightarrow\{A, B, C\}$ is a linear functional on $Q$, so may be represented in the form $\{A, B, C\}=(A \vee B, C)$ where $A \vee B$ is an element of $Q$ depending on $A$ and $B$. It is clear that $A \vee B$ is bilinear and so defines a multiplication from $Q \times Q$ to $Q$. It is easily seen that $A \vee B$ must take the form

$$
A \vee B=\frac{\sqrt{3}}{2}(A B+B A)-\frac{1}{\sqrt{3}} \operatorname{tr} A B
$$

The operation $V$ is commutative but not associative. The other algebra is 
induced by the antisymmetric third-order invariant. This induces a skew product $\wedge$ :

$$
A \wedge B=\frac{-i}{2}[A, B], \quad[A, B, C]=(A \wedge B, C) .
$$

The algebra $Q$ with product $\wedge$ is a Lie algebra.

The important physical quantities in Gell-Mann's "Eight fold way", namely the electric charge, hypercharge, etc., correspond to solutions of the equation

$$
q \vee q+\eta(q) q=0
$$

where $\eta(q)$ is a real number. A solution $q$ of this equation is an essential idempotent of the $\vee$ algebra. These essential idempotents are the directions of symmetry breaking for the adjoint representation: that is, they are the critical points of the invariant functional $\{A, A, A\}$ on the unit sphere $(A, A)=1$.

Equation (14) is mathematically identical to the reduced bifurcation equations which we have already encountered, and in fact are the $S U(3)$ equivalent of the equations which arise in the analysis of bifurcation from rotationally-invariant states. A general algebraic theory of such equations and their solutions would be extremely useful.

I regard the problem of developing algebraic algorithms for finding all solutions of (14), their stability, and their symmetry, for a general irreducible representation of a compact group, as the most important problem to be resolved in the theory of symmetry breaking.

\section{REFERENCES}

1. J. F. G. Auchmuty, Bifurcating waves, Bifurcation Theory and Applications in Scientific Disciplines (O. Gurel and O. E. Rössler, eds.) Ann. New York Acad. Sci., 316 (1979), 263-278.

2. J. F. G. Auchmuty and G. Nicholis, Dissipative structures, catastrophes, and pattern formation: a bifurcation analysis, Proc. Nat. Acad. Sci. U. S. A. 71 (1974), 2748-2751.

3. Bifurcation analysis of reaction-diffusion equations. III, Chemical ascillations, Bull. Math. Biol. 38 (1976), 325-350.

4. T. B. Benjamin, Applications of topological degree theory to problems of hydrodynamic stability, Math. Proc. Cambridge Philos. Soc. 79 (1978), 373-392.

5. _ Bifurcation phenomena in steady flows of a viscous fluid. I. Theory; II. Experiments, Proc. Roy. Soc. London Ser. A 359 (1978), 1-26; 27-43.

6. J. L. Birman, Symmetry changes, phase transitions and ferroelectricity, Ferrolectricity, Elsevier, New York, 1967.

7. F. Busse, The stability of finite amplitude cellular convection and its relation to an extremum principle, J. Fluid Mech. 30 (1967), 625-650.

8. ___ Patterns of convection in spherical shells, J. Fluid Mech. 72 (1975), 67-85.

9. Mathematical problems of dynamo theory, Applications of Bifurcation Theory (P. Rabinowitz, ed.), Academic Press, New York, 1977.

10. A. Chenciner and G. Iooss, Bifurcations de tores invariants, Arch. Rational Mech. Anal. 69 (1979), 109-198.

11. D. Coles, Transition in circular Couette flow, J. Fluid Mech. 21 (1965), 385-425.

12. J. D. Cowan and G. B. Ermentrout, Secondary bifurcation in neuronal nets, SIAM J. Appl. Math. (to appear).

13. A mathematical theory of visual hallucination patterns, Biological Cybernetics 34 (1979), 137-150.

14. M. Crandall and P. Rabinowitz, Bifurcation, perturbation of simple eigenvalues, and linearized stability, Arch. Rational Mech. Anal. 52 (1973), 161-180.

15. $\longrightarrow$ The Hopf bifurcation theorem in infinite dimensions, Arch. Rational Mech. Anal. 67 (1977), 53-72. 
16. J. Dieudonné, Foundations of modern analysis, Academic Press, New York, 1969.

17. P. R. Fenstermacher, H. L. Swinney and J. P. Gollub, Dynamical instabilities and the transition to chaotic Taylor vortex, J. Fluid Mech. 94 (1979), 103.

18. P. Fife, The Benard problem for general fluid dynamical equations and remarks on the Boussinesq approximation, Indiana Univ. Math. J. 20 (1970), 303-326.

19. __ Mathematical aspects of reacting and diffusing systems, Lecture Notes in Biomath., vol. 28, Springer-Verlag, Berlin and New York, 1979.

20. J. P. Gollub and H. L. Swinney, Onset of turbulence in a rotating fluid, Phys. Rev. Letters 35 (1975), 921.

21. M. Golubitsky and D. Schaeffer, $A$ theory for imperfect bifurcation via singularity theory, Comm. Pure Appl. Math. 32 (1979), 21-98.

22. J. Guckenheimer, The bifurcation of quadratic functions, Ann. New York Acad. Sci. 316 (1979), 78-85.

23. H. Haken, Laser theory, Handbuch der Physik, vol. XXV/2C, Springer-Verlag, Berlin, 1970.

24. M. Hénon and Y. Pomeau, Two strange attractors with a simple structure, Turbulence and Navier-Stokes Equations, (R. Temam, ed.) Lecture Notes in Math., vol. 565, Springer-Verlag, Berlin and New York, 1975.

25. D. Henry, Geometric theory of semilinear parabolic equations, Univ. of Kentucky Lecture Notes, 1974.

26. K. Hepp and E. H. Lieb, Phase transitions in reservoir-driven open systems with applications to lasers and superconductors, Helvetica Physica Acta 46 (1973), 574-603.

27. M. Herschkowitz-Kaufman and T. Erneux, The bifurcation diagram of model chemical reactions, Ann. New York Acad. Sci. 316 (1979), 296-313.

28. E. Hopf, Abzweigung liner Periodischen Lösung eines Differential Systems, Berichten der Math.-Phys. Klasse der Sächisten Akademie der Wissenschaften zu Leipzig 94 (1942), 1-22.

29. , A mathematical example displaying features of turbulence, Comm. Pure Appl. Math. 1 (1948), 303-322.

30. G. W. Hunt, Imperfection-sensitivity of semi-symmetric branching, Proc. Roy Soc. London Ser. A 357 (1977), 193-211.

31. G. Iooss, Existence et stabilité de la solution périodique secondaire intervenant dans les problèmes d'évolution du type Navier-Stokes, Arch. Rational Mech. Anal. 47 (1972), 301-329.

32. __ Bifurcation of maps and applications, North-Holland, Amsterdam, 1979.

33. G. Iooss and D. Joseph, Bifurcation and stability of $n T$ periodic solutions branching from T-periodic solutions at points of resonance, Arch. Rational Mech. Anal. 66 (1977), 135-172.

34. The behavior of solutions lying on an invariant 2-torus arising from the bifurcation of a periodic solution, Trends and Applications of Pure Mathematics to Mechanics, vol. 3, Pitman, New York, 1979

35. V. Iudovic, Stability of steady flows of viscous incompressible fluids, Soviet Physics Dokl. 10 (1965), 293-295.

36. __ On the origin of convection, Prikl. Mat. Meh. 30 (1966), 1193-1199.

37. $\longrightarrow$ On the stability of self-oscillations of a liquid, Soviet Math. Dokl. 11 (1970), $1543-1546$.

38. __ Appearance of auto-oscillations in a fluid, Prikl. Mat. Meh. 35 (1971), 638-655.

39. M. V. Jaric and J. L. Birman, New algorithms for the Molien functions, J. Math. Phys. 18 (1977), 1456-1458.

40. D. Joseph, Remarks about bifurcation and stability of quasi-periodic solutions which bifurcate from periodic solutions of the Navier-Stokes equations, Nonlinear Problems in the Physical Sciences and Biology, Lecture Notes in Math., vol. 322, Springer-Verlag, Berlin and New York, 1973.

41. D. D. Joseph, Stability of fluid motions. I, II, Springer-Verlag, Berlin and New York, 1976.

42. D. D. Joseph and D. H. Sattinger, Bifurcating time periodic solutions and their stability, Arch. Rational Mech. Anal. 45 (1972), 75-109.

43. H. Kielhöfer, Stability and semilinear evolution equations in Hilbert space, Arch. Rational Mech. Anal. 57 (1974), 150-165.

44. J. P. Keener and H. B. Keller, Perturbed bifurcation theory, Arch. Rational Mech. Anal. 50 (1973), 159-175. 
45. J. P. Keener, Perturbed bifurcation theory at multiple eigenvalues, Arch. Rational Mech. Anal. 56 (1974), 348-366.

46. K. Kirchgässner and H. Kielhöfer, Stability and bifurcation in fluid dynamics, Rocky Mountain J. Math. 3 (1973), 275-318.

47. K. Kirchgässner and P. Sorger, Branching analysis for the Taylor problem, Quart J. Mech. Appl. Math. 22 (1969), 183-209.

48. J. G. Kirkwood and E. Monroe, Statistical mechanics of fusion, J. Chem. Phys. 9 (1941), 514-526.

49. E. L. Koschmieder, Benard convection, Advances in Chem. Phys. 26 (1974), p. 177.

50. J. J. Kozak, Phase transitions as a problem in bifurcation theory, Ann. New York Acad. Sci. 316 (1979), 417-432.

51. L. Landau, On the problem of turbulence, C.R. Acad. Sci. USSR 44 (1944), 311.

52. O. E. Lanford, Bifurcation of periodic solutions into invariant tori: the work of Ruelle and Takens, Lecture Notes in Math., vol. 322, Springer-Verlag, Berlin and New York, 1973.

53. R. Larter and P. Ortoleva, Self-electophoretic phenomena, J. Theoret. Biol. (to appear).

54. N. Levinson, $A$ second order differential equation with singular solutions, Ann. of Math. 50 (1949), 127-153.

55. E. N. Lorenz, Deterministic nonperiodic flow, J. Atmospheric Sci. 20 (1963), 130-141.

56. G. Yu Lyabarskii, The application of group theory in physics, Pergamon Press, New York, 1960.

57. W. V. R. Malkus and G. Veronis, Finite amplitude cellular convection, J. Fluid Mech. 4 (1958), 225-269.

58. J. Marsden and M. McCracken, The Hopf bifurcation and its applications, Lecture Notes in Appl. Math. Sci. vol. 18, Springer-Verlag, Berlin and New York, 1976.

59. R. M. May, Bifurcations and dynamic complexity in ecological systems, Ann. New York Acad. Sci., 316 (1979), 517-529.

60. J. B. McLeod and D. H. Sattinger, Loss of stability and bifurcation at a double eigenvalue, J. Functional Anal. 14 (1973), 62-84.

61. L. Michel, Les brisures spontanées de symétrie en physique, J. Phys. 36 (1975), C7/41C7/51.

62. L. Michel and L. A. Radicati, The geometry of the octet, Ann. Inst. Henri Poincare, Sect. A 18 (1973), 185-214.

63. _ Properties of the breaking of hadronic internal symmetny, Ann. of Physics 66 (1971), 758-783.

64. W. Miller, Symmetry groups and their applications, Academic Press, New York, 1972.

65. J. Neimark, On some cases of periodic motions depending on parameters, Dokl. Akad. Nauk. SSR 129 (1959), 736-739.

66. H. G. Othmer, Applications of bifurcation theory in the analysis of spatial and temporal pattern formation, Ann. New York Acad. Sci. 316 (1979), 64-77.

67. H. G. Othmer and L. E. Scriven, Nonlinear aspects of dynamic pattern in cellular networks, J. Theoret Biol. 43 (1974), 83-112.

68. G. Prodi, Theoremi di tipo locale per il sistema di Navier-Stokes e stabilita delle soluzioni stazionari, Rend. Sem. Mat. Univ. Padova 32 (1962), 374-397.

69. P. Rabinowitz, Existence and nonuniqueness of rectangular solutions of the Bénard problem, Arch. Rational Mech. Anal. 24 (1968), 32-57.

70. H. J. Raveché and C. A. Stuart, Bifurcation of solutions with crystalline symmetry, J. Math. Phys. 17 (1976), 1949-1953.

71. E. L. Riess, Imperfect bifurcation, Applications of Bifurcation Theory (P. Rabinowitz, ed.), Academic Press, New York, 1977.

72. P. H. Roberts, Dynamo theory, Mathematical Problems in the Geophysical Sciences, Lectures in Appl. Math. vol. 14, Amer. Math. Soc., Providence, R. I., 1971.

73. O. Rössler, Continuous chaos-from prototype equations, Ann. New York Acad. Sci. 316 (1979), 376-392.

74. D. Ruelle and F. Takens, On the nature of turbulence, Comm. Math. Phys. 20 (1971), 167-192.

75. D. Ruelle, The Lorenz attractor and the problem of turbulence, Turbulence and NavierStokes Equations (R. Temam, ed.), Lecture Notes in Math., vol. 565, Springer-Verlag, Berlin and New York, 1975. 
76. R. Sacker, On invariant surfaces and bifurcation of periodic solutions of ordinary differential equations, New York University IMM-NYU, 333 (1964).

77. D. Sather, Branching of solutions of nonlinear equations, Rocky Mountain J. Math. 3 (1973), 204-250.

78. , Branching and stability for nonlinear gradient operators, Nonlinear Analysis (Cesari, Kannan, and Weinberger, eds.), Academic Press, New York, 1978.

79. D. H. Sattinger, The mathematical problem of hydrodynamic stability, J. Math. Mech. 19 (1970), 797-817.

80. _ Stability of bifurcating solutions by Leray-Schauder degree, Arch. Rational Mech. Anal. 43 (1971), 154-166.

81. _ Bifurcation of periodic solutions of the Navier-Stokes equations, Arch. Rational Mech. Anal. 41 (1971), 68-80.

82. __ Topics in stability and bifurcation theory, Lecture Notes in Math., vol. 309, Springer-Verlag, Berlin and New York, 1973.

83. __ Group representation theory and branch points of nonlinear functional equations, SIAM J. Math. Anal. 8 (1977), 179-201.

84. Group representation theory, bifurcation theory, and pattern formation, J. Functional Anal. 28 (1978), 58-101.

85. ___ Selection mechanisms for pattern formation, Arch. Rational Mech. Anal. 66 (1977), $31-42$.

86. ___ Bifurcation from rotationally invariant states, J. Math. Phys. 19 (1978), 1720-1732.

87. __ Group theoretic methods in bifurcation theory, Lecture Notes in Math., vol. 762, Springer-Verlag, Berlin and New York, 1979.

88. R. A. Schmitz, G. T. Renola and P. C. Garrigan, Observations of complex dynamic behavior in the $\mathrm{H}_{2}-\mathrm{O}_{2}$ reaction in nickel, Ann. New York Acad. Sci. 316 (1979), 638-651.

89. S. Smale, Differential dynamical systems, Bull. Amer. Math. Soc. 73 (1967), 747-817.

90. J. T. Stuart, On the nonlinear mechanics of wave disturbances in stable and unstable parallel flows, J. Fluid Mech. 9 (1960), 353-370.

91. G. I. Taylor, Stability of a viscous fluid contained between two rotating cylinders, Philos. Trans. Roy. Soc. Ser. A 223 (1923), 284-343.

92. E. E. Tareva, On a microscopic approach to the theory of polymorphic phase transitions, Physics Letters 49 A (1974), 309-310.

93. E. E. Tareva and T. I. Trapezina, The bifurcation approach to the theory of the first order isotropic-nematic phase transition, Physics Letters 60 A (1977), 217-218.

94. J. M. T. Thompson, Bifurcational aspects of catastrophe theory, Ann. New York Acad. Sci. 316 (1979), 553-571.

95. V. A. Trenogin and B. V. Loginov, The use of group properties to determine multi-parameter families of solutions of nonlinear equations, Mat. Sb. 85 (127) (1971), 440-454; English transl. Math. USSR Sbornik 14 (1971), 438-452.

96. A. M. Turing, The chemical basis of morphogenesis, Philos. Trans. Royal Soc. London Ser. B 237 (1952), 37-72.

97. M. M. Vainberg and V. A. Trenogin, The methods of Lyapounov and Schmidt in the theory of nonlinear equations and their further development, Russian Math. Surveys 17 (1962), 1-60.

98. ___ Theory of branching of solutions of nonlinear equations, Noordhoff, Leyden, 1974.

99. W. Velte, Stabilitätsverhalten und verzwergung stationäres Lösungen der Navier-Stokesschen Gleichungen, Arch. Rational Mech. Anal. 16 (1964), 97-125.

100. H. Weinberger, On the stability of bifurcating solutions, Nonlinear Analysis (Cesari, Kannan, and Weinberger, eds.), Academic Press, New York, 1978.

101. J. D. Weeks, S. A. Rice and J. J. Kozak, Analytic approach to the theory of phase transitions, J. Chem. Phys. 52 (1970), 2416-2426.

102. E. Wigner, Group theory and atomic spectra, Academic Press, New York, 1959.

School of Mathematics, University of Minnesota, Minneapolis, Minnesota 55455 
